Berardino Sciunzi · Enrico Valdinoci

JEMS

\title{
Mean curvature properties for $p$-Laplace phase transitions
}

Received December 12, 2004

\begin{abstract}
This paper deals with phase transitions corresponding to an energy which is the sum of a kinetic part of $p$-Laplacian type and a double well potential $h_{0}$ with suitable growth conditions. We prove that level sets of solutions of $\Delta_{p} u=h_{0}^{\prime}(u)$ possessing a certain decay property satisfy a mean curvature equation in a suitable weak viscosity sense. From this, we show that, if the above level sets approach uniformly a hypersurface, the latter has zero mean curvature.
\end{abstract}

Keywords. Ginzburg-Landau-Allen-Cahn phase transition models, $p$-Laplacian operator, sliding methods, geometric and qualitative properties of solutions

\section{Contents}

1. Introduction . . . . . . . . . . . . . . . . . . . . . . . . . 319

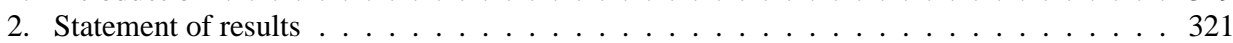

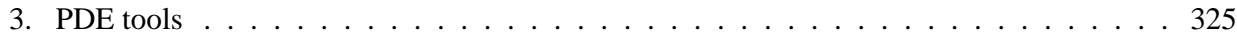

4. Technical and elementary lemmata . . . . . . . . . . . . . . . . . . 328

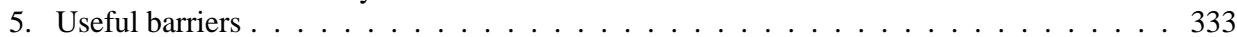

6. Sliding methods . . . . . . . . . . . . . . . . . . . . . . . . . . . . 342

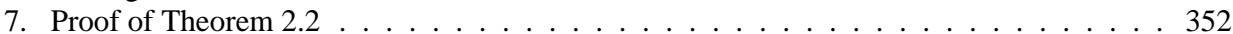

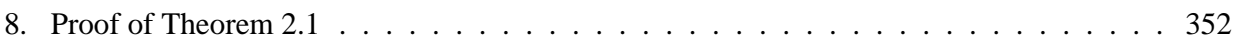

9. Comments on the decay property $(2.1)$ and proof of Theorem $2.3 \ldots \ldots \ldots 355$

\section{Introduction}

Given a domain $\Omega \subseteq \mathbb{R}^{N}$, we define the following functional on $W^{1, p}(\Omega)$ :

$$
\mathcal{F}_{\Omega}(u)=\int_{\Omega}\left(\frac{|\nabla u(x)|^{p}}{p}+h_{0}(u(x))\right) d x .
$$

In girum imus nocte et consumimur igni: we are very much indebted to Ovidiu Savin, whose magnificent work [18] has deeply inspired the present paper. This research has been supported by MIUR Variational Methods and Nonlinear Differential Equations.

B. Sciunzi, E. Valdinoci: Dipartimento di Matematica, Università di Roma Tor Vergata, Via della Ricerca Scientifica, 1, I-00133 Roma, Italy;

e-mail: sciunzi@mat.uniroma2.it, valdinoci@mat.uniroma2.it 
Here and in what follows, we suppose $1<p<\infty$ and $h_{0} \in C^{0}([-1,1]) \cap C^{1,1}((-1,1))$. We will also assume that, for some $0<c<1<C$ and some $\theta^{*} \in(0,1)$,

$$
h_{0}(\zeta)>0 \text { for any } \zeta \in(-1,1) \text {, }
$$

for any $\theta \in[0,1], \quad c \theta^{p} \leq h_{0}(-1+\theta) \leq C \theta^{p}$ and $c \theta^{p} \leq h_{0}(1-\theta) \leq C \theta^{p}$,

for any $\theta \in\left[0, \theta^{*}\right), \quad h_{0}^{\prime}(-1+\theta) \geq c \theta^{p-1}$ and $h_{0}^{\prime}(1-\theta) \leq-c \theta^{p-1}$.

Quantities depending only on the constants above will be referred to as "universal constants". We also assume a convexity property of $h_{0}$ near \pm 1 , namely that $h_{0}^{\prime}$ is ncreasing in $\left(-1,-1+\theta^{*}\right) \cup\left(1-\theta^{*}, 1\right)$.

As a model example for a potential $h_{0}$ satisfying the conditions stated above, one may consider

$$
h_{0}(\zeta):=\left(1-\zeta^{2}\right)^{p}
$$

In the literature, $h_{0}$ is often referred to as a "double well" potential, while its derivative $h_{0}^{\prime}$ is sometimes called a "bi-stable nonlinearity".

In light of the hypotheses above, with no loss of generality, possibly reducing the size of $\theta^{*}$, we may and do assume that

$$
h_{0}(\zeta) \geq \max _{\left[-1,-1+\theta^{*}\right] \cup\left[1-\theta^{*}, 1\right]} h_{0} \quad \text { for any } \zeta \in\left[-1+\theta^{*}, 1-\theta^{*}\right] .
$$

Notice that, if $u \in W^{1, p}(\Omega),|u|<1$, is critical for $\mathcal{F}_{\Omega}$, then $u$ satisfies in the weak sense the following singular/degenerate elliptic equation of $p$-Laplacian type:

$$
\Delta_{p} u(x)=h_{0}^{\prime}(u(x))
$$

for any $x \in \Omega$. Here and in what follows, we make use of the standard notation

$$
\Delta_{p} u:=\operatorname{div}\left(|\nabla u|^{p-2} \nabla u\right) .
$$

Notice also that, if $h_{0}$ can be extended to a function which is $C^{1}$ in a neighborhood of $[-1,1]$, then 1.5$)$ holds for any $u$ critical for $\mathcal{F}_{\Omega} \mathrm{sq}^{1}$ that $|u| \leq 1$.

The functional $\mathcal{F}_{\Omega}$ above has been widely studied both for pure mathematical reasons and for physical applications.

For $p=2$, the model was introduced by [7] in order to approximate the behavior of minimal surfaces and a famous conjecture concerning level sets of solutions of (1.5) was presented (see also [2], [1], [18] and [22]).

In [14] and [13] the connection between $\mathcal{F}_{\Omega}$ and minimal surfaces was developed, proving, with $\Gamma$-convergence methods, that if $u$ is a Class A minimizer for the functional (i.e., if $\mathcal{F}_{K}(u) \leq \mathcal{F}_{K}(u+\phi)$ for any compact set $K \subset \mathbb{R}^{N}$ and any $\left.\phi \in C_{0}^{\infty}(K)\right)$, and $u_{\varepsilon}(x):=u(x / \varepsilon)$, then $u_{\varepsilon}$ has a subsequence, say $u_{\varepsilon_{j}}$, which converges to a step function $\chi_{E}-\chi_{\mathbb{R}^{N} \backslash E}$, where $\partial E$ is a minimal surface (in the sense of [10]) and $\chi_{X}$ is the characteristic function of a set $X \subseteq \mathbb{R}^{N}$. A more geometric version of this result was

\footnotetext{
1 For other comments on the relation between conditions $|u| \leq 1$ and $|u|<1$, see the footnote on page 327
} 
obtained in [5], showing that the level sets $\left\{u_{\varepsilon_{j}}=0\right\}$ converge locally uniformly $\left.\right|^{2}$ to $E$. Remarkably, the proof in [5] also showed a "density estimate" property for such level sets, stating that they somehow "behave in measure as if they were codimension 1 sets" (see also [21] for further details).

The results in [14] and [13] have been extended to the case $p \neq 2$ in [4], while the ones in [5] have been extended to the case $p \neq 2$ in [15] and [16].

Some of the results of this paper can be seen as viscosity versions of the ones in [4]: indeed, we will show that if the level sets of solutions approach a limit hypersurface, then this is a zero mean curvature equation in the viscosity ${ }^{3}$ sense. Differently from [4], we are able to consider here also non-minimal solutions, replacing the minimality condition of [4] by a weaker decay assumption (see (2.1) below); the counterpart of dropping the minimality assumption in our results is that the limit surface obtained is not necessarily minimal (as in [4]), but only of zero mean curvature.

What is more, we show that level sets of solutions also enjoy a weak viscosity zero mean curvature property. Roughly speaking, we will prove that the level set $\left\{u_{\varepsilon}=0\right\}$ cannot be touched from below by a convex paraboloid in a neighborhood of the origin (which gets small with $\varepsilon$ ). In some sense, we may think that $\left\{u_{\varepsilon}=0\right\}$ attains a zero mean curvature property (though in a weak viscosity sense) even "before" converging to the limit surface. The fact that level sets inherit further properties from the minimal surface limit case is related with the flatness regularity of low dimensional level sets first conjectured by De Giorgi for $p=2$. In this setting, the weak viscosity equation fulfilled by level sets that we prove in this paper will play a decisive rôle in the forthcoming paper [22].

Independently of its importance in pure mathematics, the functional studied in this paper has also some physical relevance, since it appears in the study of the equilibrium of elastic rods under tension (see [3]) and in the van der Waals-Allen-Cahn-Hilliard and Ginzburg-Landau theories of phase transition (see, for instance, [17]). In the latter situation, the term $|\nabla u|^{p}$ in the functional represents a penalization to the total energy, which keeps under control the formation of interfaces (see [11]).

\section{Statement of results}

To state our result, we need to recall some standard convention about the sign of the mean curvature of a paraboloid. Let us consider a hyperplane $\pi \subset \mathbb{R}^{N}$ with normal vector $v$. Let $S$ be a hypersurface and $P$ a paraboloid with vertex at some point $x$, and let us assume that they are tangent to each other and to $\pi$ at $x$. We say that $P$ touches $S$ from below at $x$ in $B_{\rho}(x)$ if for any $y \in S$ and $z \in P$ with $y, z \in B_{\rho}(x)$ and $y-z$ in the same direction as $v$, we have $(y-z) \cdot v \geq 0$. An analogous definition can be given for a paraboloid touching from above.

\footnotetext{
2 For a formal definition of such locally uniform convergence, see 2.2 below.

3 In spite of the natural variational setting of the problem, viscosity solution methods for $p$ Laplacian operators are coming into fashion and they are producing quite a number of interesting results (see, for instance, [12] and the references therein). In this paper, however, there will be some interplay between viscosity and weak Sobolev formulation.
} 


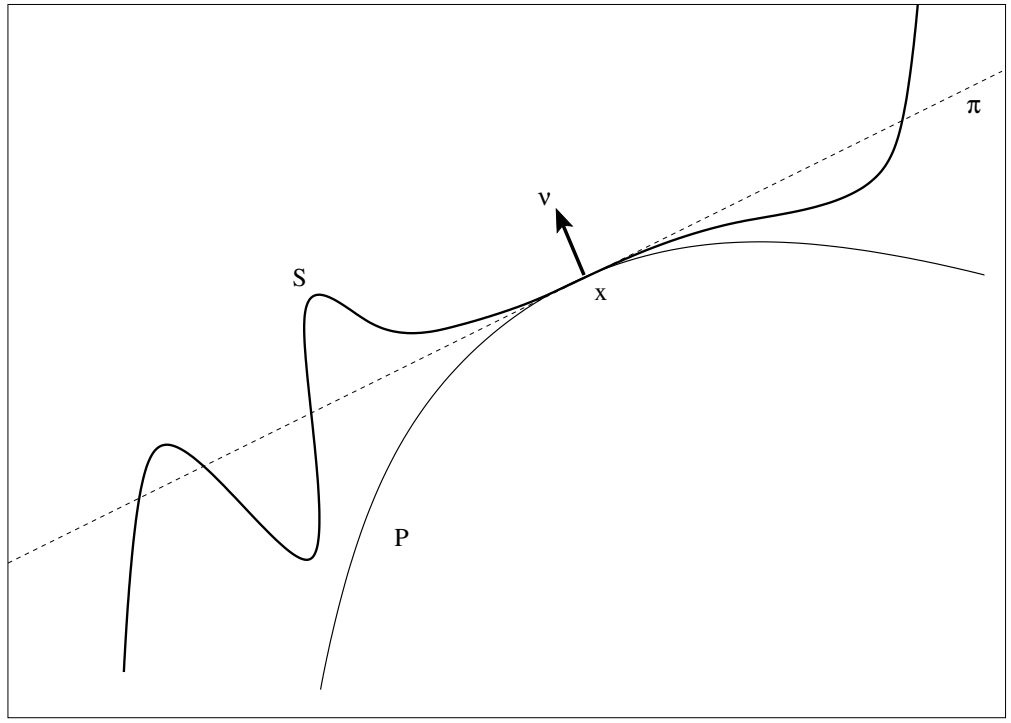

$P$ touches $S$ from below at $x$

Of course, up to a suitable choice of coordinates, one may assume that $x=0, \pi=$ $\left\{x_{N}=0\right\}$ and $v=e_{N}$. In this set of coordinates, the paraboloid $P$ takes the form ${ }^{4}$

$$
\left\{\left(x^{\prime}, x_{N}\right) \in \mathbb{R}^{N-1} \times \mathbb{R}: x_{N}=\frac{1}{2} x^{\prime} \cdot M x^{\prime}\right\}
$$

for some $M \in \operatorname{Mat}((N-1) \times(N-1))$. We say that $P$ has non-negative mean curvature if $\operatorname{tr} M \geq 0$. Analogously, one may define positive, negative, non-positive and zero mean curvature. Obviously, the sign of the mean curvature depends on the orientation of $v$, i.e., changing $v$ to $-v$ turns a positive mean curvature into a negative one, and so on. Similarly, changing $v$ to $-v$ transforms touching from below into touching from above.

The solutions $u$ of 1.5 we deal with are assumed to have the following decay property: given $\omega \in \mathrm{S}^{N-1}$, there exists a universal constant $L>0$ so that, for any $l \geq L$,

- if $\{u=0\} \cap\left\{|x-(\omega \cdot x) \omega|_{\infty} \leq l\right\} \subset\left\{\omega \cdot x \geq-c_{1}^{\star} l\right\}$, then $u(x)<-1+\theta^{*}$ for any $x=\left(x^{\prime}, x_{N}\right) \in \mathbb{R}^{N-1} \times \mathbb{R}$ satisfying

$$
\omega \cdot x<-c_{2}^{\star} l \text { and }|x-(\omega \cdot x) \omega|_{\infty}<c_{3}^{\star} l
$$

- if $\{u=0\} \cap\left\{|x-(\omega \cdot x) \omega|_{\infty} \leq l\right\} \subset\left\{\omega \cdot x \leq c_{1}^{\star} l\right\}$, then $u(x)>1-\theta^{*}$ for any $x=\left(x^{\prime}, x_{N}\right) \in \mathbb{R}^{N-1} \times \mathbb{R}$ satisfying

$$
\omega \cdot x \geq c_{2}^{\star} l \quad \text { and } \quad|x-(\omega \cdot x) \omega|_{\infty}<c_{3}^{\star} l .
$$

\footnotetext{
4 We will freely use the standard notation $x=\left(x^{\prime}, x_{N}\right) \in \mathbb{R}^{N-1} \times \mathbb{R}$ to denote a point $x \in \mathbb{R}^{N}$.
} 
Here $c_{2}^{\star}>c_{1}^{\star} \in(0,1)$ and $c_{3}^{\star} \in\left(0,1-c_{2}^{\star}\right)$ are intended to be positive constants, to be fixed once for all. For definiteness, we will take $c_{1}^{\star}:=1 / 10, c_{2}^{\star}:=1 / 5$ and $c_{3}^{\star}:=1 / 2$.

In $\$ 9$, we will give further details and motivation for assumption 2.1, showing also that it is fulfilled by Class A minimizers of $\mathcal{F}$.

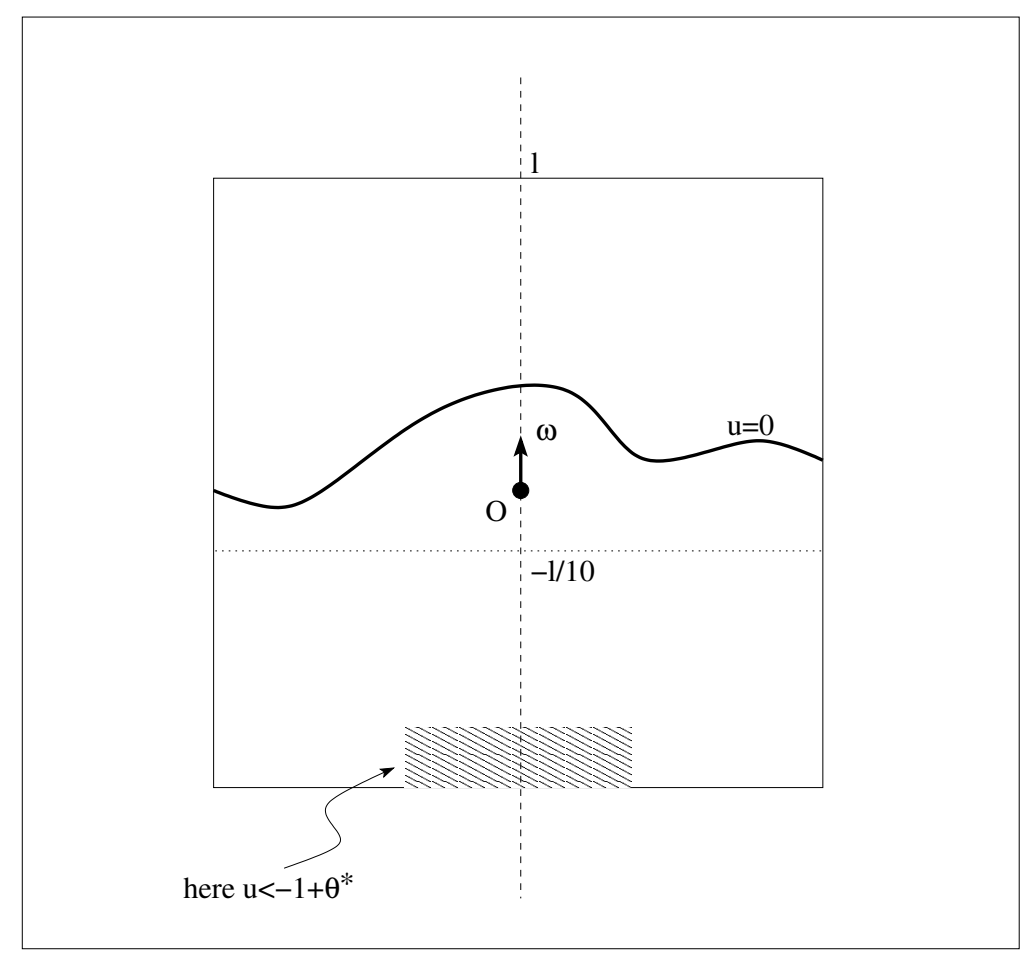

Condition 2.1

Quantities depending only on $N, p$ and on the quantities introduced in 1.2 , (1.3) will often be referred to as "universal constants". With these conventions, we are ready to state our main result:

Theorem 2.1. Let $u \in W_{\mathrm{loc}}^{1, p}\left(\mathbb{R}^{N}\right)$ be a Sobolev weak solution of 1.5 in the whole $\mathbb{R}^{N}$ satisfying 2.1), with $|u| \leq 1$. Let $\mathcal{S}=\partial \mathcal{E}$ be a continuous hypersurface in $\mathbb{R}^{N}$. Let $u_{\varepsilon}(x):=u(x / \varepsilon)$. Assume that $u_{\varepsilon}$ converges in $L_{\text {loc }}^{1}$ to $\chi_{\mathcal{E}}-\chi_{\mathbb{R}^{N} \backslash \mathcal{E}}$ and that $\left\{u_{\varepsilon}=0\right\}$ converges locally uniformly to $\mathcal{S}$, i.e., for any compact set $K \subset \mathbb{R}^{N}$,

$$
\lim _{\varepsilon \rightarrow 0} \sup _{x \in\left\{u_{\varepsilon}=0\right\} \cap K} \operatorname{dist}(x, \mathcal{S})=0 .
$$

Then $\mathcal{S}$ satisfies the zero mean curvature equation in the viscosity sense. 
More explicitly, let $x^{\star} \in \mathcal{S}$ be so that, for any $r>0$,

$$
\mathfrak{L}\left(B_{r}\left(x^{\star}\right) \cap\left(\mathbb{R}^{N} \backslash \mathcal{E}\right)\right)>0 \quad \text { and } \quad \mathfrak{L}\left(B_{r}\left(x^{\star}\right) \cap \mathcal{E}\right)>0,
$$

where $\mathfrak{L}$ denotes the $N$-dimensional Lebesgue measure. Assume also that $\mathcal{S}$ admits a tangent hyperplane in $x^{\star}$. Then:

- if a paraboloid with vertex at $x^{\star}$ touches $\mathcal{S}$ from below at $x^{\star}$, then its mean curvature at $x^{\star}$ must be non-positive;

- if a paraboloid with vertex at $x^{\star}$ touches $\mathcal{S}$ from above at $x^{\star}$, then its mean curvature at $x^{\star}$ must be non-negative.

In particular, if $x^{\star} \in \mathcal{S}$ is so that $\mathcal{S}$ is $C^{2}$ in a neighborhood of $x^{\star}$, then the mean curvature of $\mathcal{S}$ at $x^{\star}$ is zero in the classical sense.

The proof deeply relies on the ideas of [18], which deals with the case $p=2$, and it is geometric in nature. The technique presented seems very flexible, and it may also be appropriate for further interesting extensions (such as more general functionals related with non-flat metrics on manifolds or fluid dynamics, operators in non-divergence form, fully nonlinear equations, etc.). In the case $p \neq 2$, some additional care is needed in the sliding procedure, since, due to the singularity or degeneracy of the $p$-Laplacian operator at points where $\nabla u$ vanishes, no general maximum and comparison results are available in the literature. Also, a careful choice of parameters is necessary to deal with the more severe nonlinearities provided by the $p$-Laplace equation.

Theorem 2.1 will follow from a stronger result concerning a mean curvature property, in a weak viscosity sense, for level sets of rescaled solutions. That result is the following:

Theorem 2.2. Let $u \in W_{\mathrm{loc}}^{1, p}\left(\mathbb{R}^{N}\right)$ be a Sobolev weak solution of 1.5 in the whole $\mathbb{R}^{N}$, satisfying (2.1) for $\omega=e_{N}$, so that $|u| \leq 1$ and $u(0)=0$. Let $\mathfrak{d} \in(0,1)$ and $M \in$ $\operatorname{Mat}((N-1) \times(N-1))$ with

$$
\operatorname{tr} M>\mathfrak{d}\|M\| \quad \text { and } \quad\|M\| \leq \mathfrak{d}^{-1} .
$$

Let $u_{\varepsilon}(x):=u(x / \varepsilon)$ and

$$
\Gamma:=\left\{x=\left(x^{\prime}, x_{N}\right) \in \mathbb{R}^{N-1} \times \mathbb{R}: x_{N}=\frac{1}{2} x^{\prime} \cdot M x^{\prime}\right\} .
$$

Then there exist a universal $\mathfrak{d}^{\star}>0$ and a function $\sigma_{0}:(0,1) \rightarrow(0,1)$ such that if $\varepsilon \in\left(0, \sigma_{0}(\mathfrak{d})\right)$ and $\mathfrak{d} \in\left(0, \mathfrak{d}^{\star}\right)$, then $\Gamma$ cannot touch $\left\{u_{\varepsilon}=0\right\}$ from below in $B_{\mathfrak{d} \sqrt{\varepsilon} / \sqrt{\operatorname{tr} M}}$; more explicitly,

$$
\left\{u_{\varepsilon}=0\right\} \cap\left\{x_{N}<\frac{1}{2} x^{\prime} \cdot M x^{\prime}\right\} \cap\left\{|x|<\frac{\mathfrak{d} \sqrt{\varepsilon}}{\sqrt{\operatorname{tr} M}}\right\} \neq \varnothing .
$$

We remark that Class A minimizers of $\mathcal{F}$ are particular solutions satisfying the assumptions of Theorems 2.1 and 2.2 (provided $h_{0}$ admits a $C^{1}$ extension in a neighborhood of $[-1,1])$; thus the above theorems imply the following result: 
Theorem 2.3. Assume that $h_{0}$ admits a $C^{1}$ extension in a neighborhood of $[-1,1]$ and let $u$ be a Class A minimizer of $\mathcal{F}$ with $|u| \leq 1$. Then the claims of Theorems 2.1 and 2.2 hold true. More precisely, for $u_{\varepsilon}(x):=u(x / \varepsilon)$, the following results hold:

- If $u_{\varepsilon}$ converges in $L_{\mathrm{loc}}^{1}$ to $\chi_{\mathcal{E}}-\chi_{\mathbb{R}^{N} \backslash \mathcal{E}}$ and $\left\{u_{\varepsilon}=0\right\}$ converges locally uniformly to $\mathcal{S}$, where $\mathcal{S}=\partial \mathcal{E}$ is a continuous hypersurface in $\mathbb{R}^{N}$, then $\mathcal{S}$ satisfies the zero mean curvature equation in the viscosity sense.

- Let $u(0)=0, \mathfrak{d} \in(0,1)$ and $M \in \operatorname{Mat}((N-1) \times(N-1))$ with

$$
\operatorname{tr} M>\mathfrak{d}\|M\| \text { and }\|M\| \leq \mathfrak{d}^{-1}
$$

and let

$$
\Gamma:=\left\{x=\left(x^{\prime}, x_{N}\right) \in \mathbb{R}^{N-1} \times \mathbb{R}: x_{N}=\frac{1}{2} x^{\prime} \cdot M x^{\prime}\right\} .
$$

Then there exist a universal $\mathfrak{d}^{\star}>0$ and a function $\sigma_{0}:(0,1) \rightarrow(0,1)$ such that if $\varepsilon \in\left(0, \sigma_{0}(\mathfrak{d})\right)$ and $\mathfrak{d} \in\left(0, \mathfrak{d}^{\star}\right)$, then $\Gamma$ cannot touch $\left\{u_{\varepsilon}=0\right\}$ from below in $B_{\mathfrak{d} \sqrt{\varepsilon} / \sqrt{\operatorname{tr} M}}$.

The paper is organized as follows. In $\$ 3$ we recall some standard PDE notions, such as viscosity solutions and some comparison/maximum principles that will be of use in this paper. To make the proofs of the main results more readable, we collected some technical lemmata, mostly elementary in nature, in $\$ 4$ Of course, the expert reader may skip $\$ 3$ and $\$ 4$ and dedicate himself to the proofs of the main results, the core of which is contained in $\$ 5$ and $\$ 6$. In particular, $\$ 5$ is devoted to the construction of suitable barriers, built via the one-dimensional solution, which will be used in $\$ 6$ for the sliding method. That geometric construction is an extension of the one presented in [18]. The proof of Theorem 2.2 will be completed in $\$ 7$, while the one of Theorem 2.1 is in $\$ 8$ In $\$ 9$ we make comments on the assumption in (2.1) and prove Theorem 2.3

\section{PDE tools}

We recall here the definition of viscosity supersolution (and subsolution, and solution) for $p$-Laplacian type operators. Roughly, the notion of viscosity supersolution requires a pointwise evaluation of the $p$-Laplacian of smooth functions touching from below. However, since the $p$-Laplacian diverges at critical points for $1<p<2$, we need to exclude this case from the following definition:

Definition 3.1. Let $\Omega \subseteq \mathbb{R}^{N}$ be an open domain and let $u \in C^{0}(\Omega)$. If $p \geq 2$, we say that $u$ is a viscosity supersolution of 1.5 (or that $\Delta_{p} u \leq h_{0}^{\prime}(u)$ in the viscosity sense) if, whenever $x_{0} \in \Omega$ and $\phi \in C^{2}(\Omega)$ are such that $u\left(x_{0}\right)=\phi\left(x_{0}\right)$ and $u(x) \geq \phi(x)$ in $\Omega$, we have

$$
\Delta_{p} \phi\left(x_{0}\right) \leq h_{0}^{\prime}\left(\phi\left(x_{0}\right)\right) .
$$

If $1<p<2$, we say that $u$ is a viscosity supersolution of $(1.5)$ if, whenever $x_{0} \in \Omega$ and $\phi \in C^{2}(\Omega)$ are such that $\nabla \phi\left(x_{0}\right) \neq 0, u\left(x_{0}\right)=\phi\left(x_{0}\right)$ and $u(x) \geq \phi(x)$ in $\Omega$, we have

$$
\Delta_{p} \phi\left(x_{0}\right) \leq h_{0}^{\prime}\left(\phi\left(x_{0}\right)\right) \text {. }
$$


Analogously, if $p \geq 2$, we say that $u$ is a viscosity subsolution of (1.5) (or that $\Delta_{p} u \geq$ $h_{0}^{\prime}(u)$ in the viscosity sense) if, whenever $x_{0} \in \Omega$ and $\phi \in C^{2}(\Omega)$ are such that $u\left(x_{0}\right)=$ $\phi\left(x_{0}\right)$ and $u(x) \leq \phi(x)$ in $\Omega$, we have

$$
\Delta_{p} \phi\left(x_{0}\right) \geq h_{0}^{\prime}\left(\phi\left(x_{0}\right)\right)
$$

if $1<p<2$, we say that $u$ is a viscosity subsolution of $(1.5)$ if, whenever $x_{0} \in \Omega$ and $\phi \in C^{2}(\Omega)$ are such that $\nabla \phi\left(x_{0}\right) \neq 0, u\left(x_{0}\right)=\phi\left(x_{0}\right)$ and $u(x) \leq \phi(x)$ in $\Omega$, we have

$$
\Delta_{p} \phi\left(x_{0}\right) \geq h_{0}^{\prime}\left(\phi\left(x_{0}\right)\right)
$$

If $u$ is both a supersolution and a subsolution in the viscosity sense, we say that $u$ is a viscosity solution.

Of course, if $u \in C^{2}(\Omega)$ and $p \geq 2$, then $u$ is a viscosity solution of 1.5 if, and only if,

$$
|\nabla u|^{p-4}\left(|\nabla u|^{2} \Delta u+(p-2)\left\langle D^{2} u \nabla u, \nabla u\right\rangle\right)=h_{0}^{\prime}(u)
$$

pointwise. However, if $1<p<2$, the expression above may be ill defined even for smooth functions, due to the vanishing of the gradient. Therefore, if $1<p<2$, for a function $u \in C^{2}(\Omega)$, being a viscosity solution of $\sqrt{1.5}$ is equivalent to 3.1 holding at points where $\nabla u \neq 0$.

One of the greatest difficulties when dealing with $p$-Laplace equations is that the solutions belong generally only to the class $C^{1, \alpha}$ with $\alpha \in(0,1)$ (see [8] and [20]). Also, the $p$-Laplace operator is singular or degenerate elliptic (respectively if $1<p<2$ or $p>2$ ). A consequence of such pathologies is that there is no general comparison theorem for solutions in case $p \neq 2$. Therefore, no complete analogy is possible, in general, between the cases $p=2$ and $p \neq 2$.

In this paper, we will need to compare weak Sobolev solutions of (1.5) with viscosity supersolutions of (1.5). Even if there are no general results in the literature dealing with this problem, we will succeed in doing this by applying some results obtained in [6], together with Hopf's Lemma for $p$-Laplace equations [23], and thanks to some geometric properties of the barriers that we will introduce.

We now recall the maximum and comparison principles needed in our proofs. First of all, in [6] (see, in particular, Theorem 1.4 there) the following result is obtained:

Theorem 3.2 (Strong Comparison Principle I). Let $\Omega$ be an open (not necessarily bounded or connected) subset of $\mathbb{R}^{N}$, and let $\Lambda \in \mathbb{R}$ and $u, v \in C^{1}(\Omega)$ satisfy

$$
-\Delta_{p} u+\Lambda u \leq-\Delta_{p} v+\Lambda v, \quad u \leq v \quad \text { in } \Omega
$$

Define $Z_{u, v}=\{x \in \Omega:|D u(x)|+|D v(x)|=0\}$ if $p \neq 2, Z_{u, v}=\emptyset$ if $p=2$. If $x_{0} \in \Omega \backslash Z_{u, v}$ and $u\left(x_{0}\right)=v\left(x_{0}\right)$, then $u=v$ in the connected component of $\Omega \backslash Z_{u, v}$ containing $x_{0}$. 
An easy consequence of the above result is the following one, which will be suitable for our applications 5 .

Corollary 3.3 (Strong Comparison Principle II). Let $\Omega$ be an open (not necessarily bounded or connected) subset of $\mathbb{R}^{N}$, and let $u, v \in C^{1}(\Omega)$ satisfy

$$
-\Delta_{p} u+f(u) \leq-\Delta_{p} v+f(v), \quad u \leq v \quad \text { in } \Omega,
$$

with $f$ locally Lipschitz continuous. Define $Z_{u, v}$ as in Theorem 3.2 Then the conclusion of Theorem 3.2 holds.

Proof. Let $\epsilon>0$ be so that $B_{\epsilon}\left(x_{0}\right) \subset \Omega$ and let

$$
M_{u, v}=\max \left\{|u|_{L^{\infty}\left(B_{\epsilon}\left(x_{0}\right)\right)},|v|_{L^{\infty}\left(B_{\epsilon}\left(x_{0}\right)\right)}\right\}, \quad \Lambda=\sup _{\substack{U \neq V \\|U|,|V| \leq M_{u, v}}} \frac{|f(U)-f(V)|}{|U-V|} .
$$

Then

$$
\begin{aligned}
-\Delta_{p} u+\Lambda u & \leq-\Delta_{p} v+f(v)-f(u)+\Lambda u \leq-\Delta_{p} v+\Lambda|v-u|+\Lambda u \\
& =-\Delta_{p} v+\Lambda(v-u)+\Lambda u=-\Delta_{p} v+\Lambda v
\end{aligned}
$$

hence the result follows from Theorem 3.2

We recall that a similar result was proved in [19], under stronger assumptions.

We now state a result which will allow us to take care of the points at which solutions of (1.5) may have a vanishing gradient. To this end, we recall the following version of a more general result proved ${ }^{6}$ in [23]:

5 As a technical remark, we point out that we will use Corollary 3.3 on pages 343 and 345 , with $f:=h_{0}^{\prime}$. In our case, $h_{0}$ is not assumed to be $C^{1,1}$ in the closed interval $[-1,1]$, but only in $(-1,1)$. Nevertheless, we will be able to exclude touching points at \pm 1 with a direct argument, hence we will apply Corollary 3.3 in the domain where $h_{0}$ is $C^{1,1}$.

6 Although we do not explicitly assume $|u|<1$ here, but only $|u| \leq 1$, we think it is appropriate to notice that, in many cases of interest, the two conditions are equivalent, thanks to the results mentioned. Indeed, the condition $|u|<1$, under suitable assumptions, is fulfilled by any solution $u$ such that $|u| \leq 1$ with $|u|$ not identically equal to 1 . For instance, assume that for any $\theta \in\left[0, \theta^{*}\right)$, $h_{0}^{\prime}(-1+\theta) \leq c^{\prime} \theta^{p-1}$ and $h_{0}^{\prime}(1-\theta) \geq-c^{\prime} \theta^{p-1}$ (this condition is in particular satisfied by $\left.h_{0}(\zeta):=\left(1-\zeta^{2}\right)^{p}\right)$. Suppose there are points where $u=1$ (the case $u=-1$ follows in the same way). Since $|u|$ is not identically 1 , there exists a ball $B_{r}(x)$ with $u<1$ in int $B_{r}(x)$ and $u(y)=1$ for some $y \in \partial B_{r}(x)$. Then $w:=1-u$ is not identically zero in int $B_{r}(x)$ and $w(y)=0$. Moreover,

$$
-\Delta_{p} w=h_{0}^{\prime}(u)=\frac{h_{0}^{\prime}(1-w)}{w^{p-1}} w^{p-1} \geq-c^{\prime} w^{p-1},
$$

i.e. $-\Delta_{p} w+c^{\prime} w^{p-1} \geq 0$ weakly. We can therefore exploit the Strong Maximum Principle of Theorem 3.4 and prove that $u<1$. In the same way we also get $u>-1$. 
Theorem 3.4 (Strong Maximum Principle and Hopf's Lemma). Let $\Omega$ be an open connected (not necessarily bounded) set in $\mathbb{R}^{N}$ and suppose that $u \in C^{1}(\Omega), u \geq 0$ in $\Omega$, weakly solves

$$
-\Delta_{p} u+c u^{q}=g \geq 0 \quad \text { in } \Omega
$$

with $q \geq p-1, c \geq 0$ and $g \in L_{\mathrm{loc}}^{\infty}(\Omega)$. If $u$ is not identically zero, then $u>0$ in $\Omega$. Moreover, for any point $x_{0} \in \partial \Omega$ where the interior sphere condition is satisfied, and such that $u$ is $C^{1}$ in a neighborhood of $\Omega \cup\left\{x_{0}\right\}$ and $u\left(x_{0}\right)=0$, we have $\partial u / \partial s>0$ for any inward directional derivative.

Remark 3.5. The proof of Theorem 3.4 follows at once by applying Theorem 5 of [23] with $\beta(s)=c s^{q}, q \geq p-1$ and $c \geq 0$. In particular, the condition $q \geq p-1$ ensures that conditions (13) and (13') in [23] are fulfilled. Moreover, the condition $c \geq 0$ causes $\beta$ to be nondecreasing with $\beta(0)=0$.

\section{Technical and elementary lemmata}

This section, which may be skipped by the expert reader, collects some elementary lemmata that will be of use in the course of the proofs of the main results.

Lemma 4.1. There exists a positive constant $C$, depending only on $p$, such that

$$
a^{1 / p}-b^{1 / p} \leq C \frac{a-b}{a^{(p-1) / p}+b^{(p-1) / p}},
$$

for any $a \geq b \geq 0, a \neq 0$.

Proof. For $t \in[0,1)$, set

$$
f(t):=\frac{\left(1-t^{1 / p}\right)\left(1+t^{(p-1) / p}\right)}{1-t} .
$$

Notice that $f \in C^{0}([0,1)), f(0)=1$ and

$$
\lim _{t \rightarrow 1} f(t)=\frac{2}{p} .
$$

Hence, $f(t) \leq C$ for any $t \in[0,1)$ and so

$$
\frac{\left(a^{1 / p}-b^{1 / p}\right)\left(a^{(p-1) / p}+b^{(p-1) / p}\right)}{a-b}=f(b / a) \leq C .
$$

Lemma 4.2. For any $0 \leq s \leq t \leq \theta^{*}$,

$$
h_{0}(-1+t)-h_{0}(-1+s) \geq c\left(t^{p}-s^{p}\right)
$$

for a suitable universal constant $c>0$. 
Proof. From 1.3,

$$
h_{0}(-1+t)-h_{0}(-1+s)=\int_{s}^{t} h_{0}^{\prime}(-1+\theta) d \theta \geq \mathrm{const} \int_{s}^{t} \theta^{p-1} d \theta,
$$

which implies the desired result.

Lemma 4.3. There exists a positive constant $\tilde{C}$, depending only on $p$, so that

$$
\int_{-1+\mathfrak{b}}^{0} \frac{d \zeta}{\left((1+\zeta)^{p}-\mathfrak{a}^{p}\right)^{1 / p}} \leq \tilde{C}\left(1+\log \frac{1}{\mathfrak{b}}\right)
$$

for any $0<\mathfrak{a} \leq \mathfrak{b} \leq 1$.

Proof. Let

$$
\mathfrak{x}:= \begin{cases}1 & \text { if } \mathfrak{b}<1 / 2, \\ 0 & \text { if } \mathfrak{b} \geq 1 / 2 .\end{cases}
$$

Using the substitution $\tau:=(1+\zeta) / \mathfrak{b}$, we bound the integral above by

$$
\begin{aligned}
\int_{1}^{1 / \mathfrak{b}} \frac{d \tau}{\left(\tau^{p}-(\mathfrak{a} / \mathfrak{b})^{p}\right)^{1 / p}} & \leq \int_{1}^{1 / \mathfrak{b}} \frac{d \tau}{\left(\tau^{p}-1\right)^{1 / p}} \\
& \leq \int_{1}^{2} \frac{d \tau}{\left(\tau^{p}-1\right)^{1 / p}}+\mathfrak{x} \int_{2}^{1 / \mathfrak{b}} \frac{d \tau}{\left(\tau^{p}-1\right)^{1 / p}} .
\end{aligned}
$$

Noticing that

$$
\tau^{p}-1 \geq \frac{2^{p}-1}{2^{p}} \tau^{p}
$$

if $\tau \geq 2$ and

$$
\tau^{p}-1 \geq \tau-1
$$

if $\tau \geq 1$, we bound the quantity above by

$$
\text { const }\left(\int_{1}^{2} \frac{d \tau}{(\tau-1)^{1 / p}}+\int_{2}^{1 / \mathfrak{b}} \frac{d \tau}{\tau}\right) \leq \operatorname{const}\left(1+\log \frac{1}{2 \mathfrak{b}}\right)
$$

which proves the desired result.

Lemma 4.4. Let $U$ be an open subset of $\mathbb{R}$. Let $g \in C^{2}(U)$ and assume that $g$ has no critical points. Define

$$
\Psi^{y, l}(x):=g(|x-y|-l) .
$$

Then, for $t=|x-y|-l \in U$ and $x \neq y$, we have

$$
\Delta_{p}\left(\Psi^{y, l}(x)\right)=(p-1) g^{\prime \prime}(t)\left(g^{\prime}(t)\right)^{p-2}+\left(g^{\prime}(t)\right)^{p-1} \frac{N-1}{|x-y|} .
$$


Proof. Note that

and

$$
\nabla \Psi^{y, l}(x)=g^{\prime}(t) \frac{x-y}{|x-y|}
$$

$$
\Psi_{i j}^{y, l}(x)=g^{\prime \prime}(t) \frac{\left(x_{i}-y_{i}\right)\left(x_{j}-y_{j}\right)}{|x-y|^{2}}+g^{\prime}(t)\left(\frac{\delta_{i j}}{|x-y|}-\frac{\left(x_{i}-y_{i}\right)\left(x_{j}-y_{j}\right)}{|x-y|^{3}}\right) .
$$

Since

$$
\begin{aligned}
& \Delta_{p}\left(\Psi^{y, l}(x)\right) \\
& \quad=\left|\nabla \Psi^{y, l}(x)\right|^{p-2} \Delta \Psi^{y, l}(x)+(p-2)\left|\nabla \Psi^{y, l}(x)\right|^{p-4} \Psi_{i j}^{y, l}(x) \Psi_{i}^{y, l}(x) \Psi_{j}^{y, l}(x),
\end{aligned}
$$

we get

$$
\begin{aligned}
\Delta_{p}\left(\Psi^{y, l}(x)\right)= & \left(g^{\prime}(t)\right)^{p-2} g^{\prime \prime}(t)+(p-2) \sum_{i j}\left(g^{\prime}(t)\right)^{p-2} g^{\prime \prime}(t) \frac{\left(x_{i}-y_{i}\right)^{2}\left(x_{j}-y_{j}\right)^{2}}{|x-y|^{4}} \\
& +\left(g^{\prime}(t)\right)^{p-1} \frac{N}{|x-y|}+(p-2)\left(g^{\prime}(t)\right)^{p-1} \frac{1}{|x-y|} \\
& -\left(g^{\prime}(t)\right)^{p-1} \frac{1}{|x-y|}-(p-2) \sum_{i j}\left(g^{\prime}(t)\right)^{p-1} \frac{\left(x_{i}-y_{i}\right)^{2}\left(x_{j}-y_{j}\right)^{2}}{|x-y|^{5}} \\
= & (p-1)\left(g^{\prime}(t)\right)^{p-2} g^{\prime \prime}(t)+\left(g^{\prime}(t)\right)^{p-1} \frac{N-1}{|x-y|} .
\end{aligned}
$$

Lemma 4.5. Let $U$ and $g$ be as in Lemma 4.4. Let $\Gamma$ be a smooth hypersurface in $\mathbb{R}^{N}$ and let $d_{\Gamma}(x)$ be the signed distance function to $\Gamma$. Suppose that if $x \in \Omega$ then $d_{\Gamma}(x) \in U$. Then

$$
\Delta_{p} g\left(d_{\Gamma}(x)\right)=(p-1)\left|g^{\prime}\left(d_{\Gamma}(x)\right)\right|^{p-2} g^{\prime \prime}\left(d_{\Gamma}(x)\right)+\left|g^{\prime}\left(d_{\Gamma}(x)\right)\right|^{p-2} g^{\prime}\left(d_{\Gamma}(x)\right) \Delta d_{\Gamma}(x) .
$$

Proof. Easy calculations show that

$$
\nabla g\left(d_{\Gamma}(x)\right)=g^{\prime}\left(d_{\Gamma}(x)\right) \nabla d_{\Gamma}(x)
$$

and

$$
g_{i j}\left(d_{\Gamma}(x)\right)=g^{\prime \prime}\left(d_{\Gamma}(x)\right)\left(d_{\Gamma}\right)_{i}(x)\left(d_{\Gamma}\right)_{j}(x)+g^{\prime}\left(d_{\Gamma}(x)\right)\left(d_{\Gamma}\right)_{i j}(x) .
$$

By rotation invariance, it is not restrictive to consider a coordinate system for which

$$
\nabla d_{\Gamma}(x)=(0,0, \ldots, 0,1)
$$

and

$$
D^{2} d_{\Gamma}=\operatorname{diag}\left(\frac{-k_{1}}{1-d_{\Gamma} k_{1}}, \ldots, \frac{-k_{N-1}}{1-d_{\Gamma} k_{N-1}}, 0\right) \in \operatorname{Mat}(N \times N),
$$

where the $k_{i}$ 's are the principal curvatures of $\Gamma$ at the point where the distance is realized (see, for instance, $\$ 14.6$ of [9] for details). Therefore, taking into account [4.2] and 4.3], we get

$$
\Delta_{p} g\left(d_{\Gamma}(x)\right)=(p-1)\left|g^{\prime}\left(d_{\Gamma}(x)\right)\right|^{p-2} g^{\prime \prime}\left(d_{\Gamma}(x)\right)+\left|g^{\prime}\left(d_{\Gamma}(x)\right)\right|^{p-2} g^{\prime}\left(d_{\Gamma}(x)\right) \Delta d_{\Gamma}(x) .
$$


Lemma 4.6. Let $I \ni 0$ be an interval of $\mathbb{R}$ and let $h \in C^{1}(I)$ satisfy $h(s)>0$ for any $s \in I$. Let

$$
H(s):=\int_{0}^{s} \frac{(p-1)^{1 / p} d \zeta}{(p h(\zeta))^{1 / p}}, \quad \forall s \in I .
$$

Define also $g$ as the inverse of $H$, that is, $g(t):=H^{-1}(t)$ for any $t \in H(I)$. Then $g \in C^{2}(H(I))$ and

$$
g^{\prime}(t)=\left(\frac{p}{p-1} h(g(t))\right)^{1 / p}, \quad g^{\prime \prime}(t)=\frac{(p h(g(t)))^{(2-p) / p}}{(p-1)^{2 / p}} h^{\prime}(g(t)),
$$

for any $t \in H(I)$.

Proof. The first identity follows easily by differentiating $H(g(t))=t$. For the second claim, notice that using the first identity twice gives

$$
\begin{aligned}
g^{\prime \prime}(t) & =\frac{d}{d t}\left(\frac{p}{p-1} h(g(t))\right)^{1 / p}=\frac{(p h(g(t)))^{1 / p-1}}{(p-1)^{1 / p}} h^{\prime}(g(t)) g^{\prime}(t) \\
& =\frac{(p h(g(t)))^{2 / p-1}}{(p-1)^{2 / p}} h^{\prime}(g(t)) .
\end{aligned}
$$

Lemma 4.7. Let $\Omega$ be an open domain in $\mathbb{R}^{N}$ and let $x_{0} \in \Omega$. Let $w \in C^{1}(\Omega)$ and $v:=\nabla w\left(x_{0}\right)$. Assume that there exists $\varpi \in \mathbb{R}^{N} \backslash\{0\}$ such that

$$
w\left(x_{0}+x\right) \leq v \cdot x+w\left(x_{0}\right)
$$

for any $x \in \mathbb{R}^{N}$ so that $x+x_{0} \in \Omega$ and $\varpi \cdot x \geq 0$. If $P \in C^{2}(\Omega)$ is a quadratic function touching $w$ from below at $x_{0}$, then $\Delta_{p} P\left(x_{0}\right) \leq 0$ in the viscosity sense. Analogously, if

$$
w\left(x_{0}+x\right) \geq v \cdot x+w\left(x_{0}\right)
$$

for any $x \in \mathbb{R}^{N}$ so that $x+x_{0} \in \Omega$ and $\varpi \cdot x \geq 0$, and $P \in C^{2}(\Omega)$ is a quadratic function touching $w$ from above at $x_{0}$, then $\Delta_{p} P\left(x_{0}\right) \geq 0$ in the viscosity sense.

Proof. We prove the first claim, the second one being analogous. Since $P$ touches $w$ at $x_{0}$, $w \in C^{1}(\Omega)$ and $\nabla w\left(x_{0}\right)=v$, we have

$$
P(x)=\frac{1}{2} M\left(x-x_{0}\right) \cdot\left(x-x_{0}\right)+v \cdot\left(x-x_{0}\right)+w\left(x_{0}\right)
$$

for some $M \in \operatorname{Mat}(N \times N)$. Notice that $M$ must be non-positive definite: indeed, if $M$ had a strictly positive eigenvalue $\lambda$ with corresponding eigenvector $e$ and $|e|=1$, possibly changing $e$ into $-e$ we may assume that $\varpi \cdot e \geq 0$ and therefore, for a small $\varepsilon>0$,

$$
\begin{aligned}
\varepsilon v \cdot e+w\left(x_{0}\right) & \geq w\left(x_{0}+\varepsilon e\right) \geq P\left(x_{0}+\varepsilon e\right)=\frac{\varepsilon^{2}}{2} M e \cdot e+\varepsilon v \cdot e+w\left(x_{0}\right) \\
& \geq \frac{\varepsilon^{2} \lambda}{2}+\varepsilon v \cdot e+w\left(x_{0}\right)>\varepsilon v \cdot e+w\left(x_{0}\right),
\end{aligned}
$$


which is a contradiction. Hence, $M$ is non-positive definite and thus $\Delta P=\operatorname{tr} M \leq 0$. Now, if $p \geq 2$,

$$
\begin{aligned}
\Delta_{p} P & =|\nabla P|^{p-4}\left(|\nabla P|^{2} \Delta P+(p-2)\left\langle D^{2} P \nabla P, \nabla P\right\rangle\right) \\
& =|v|^{p-4}\left(|v|^{2} \operatorname{tr} M+(p-2) M v \cdot v\right) \leq 0
\end{aligned}
$$

at $x_{0}$, which proves the claim for $p \geq 2$.

Now assume $1<p<2$. If $v=0$, then by Definition 3.1 there is nothing to check; we may therefore assume $v \neq 0$. Let $\lambda_{1} \leq \cdots \leq \lambda_{N} \leq 0$ be the eigenvalues of $M$. Then

$$
\begin{aligned}
\Delta_{p} P & =\frac{1}{|v|^{2-p}}\left(\operatorname{tr} M-(2-p) M \frac{v}{|v|} \cdot \frac{v}{|v|}\right) \leq \frac{1}{|v|^{2-p}}\left(\lambda_{1}+\cdots+\lambda_{N}-(2-p) \lambda_{1}\right) \\
& =\frac{1}{|v|^{2-p}}\left((p-1) \lambda_{1}+\lambda_{2}+\cdots+\lambda_{N}\right) \leq 0
\end{aligned}
$$

at $x_{0}$, which ends the proof for $1<p<2$.

Next, we point out an easy property of the signed distance function to paraboloids:

Lemma 4.8. Let $M \in \operatorname{Mat}((N-1) \times(N-1))$ and $V \in \mathbb{R}^{N-1}$. Define the paraboloid

$$
\Gamma:=\left\{x=\left(x^{\prime}, x_{n}\right) \in \mathbb{R}^{N-1} \times \mathbb{R}: x_{N}=\frac{1}{2} x^{\prime} \cdot M x^{\prime}+V \cdot x^{\prime}\right\} .
$$

Let $d_{\Gamma}$ be the signed distance to $\Gamma$, with the sign convention that $d_{\Gamma} \geq 0$ above $\rrbracket^{7} \Gamma$. Then, for any $\tau \geq 0$,

$$
d_{\Gamma}\left(x+\tau e_{N}\right) \geq d_{\Gamma}(x) .
$$

Proof. Two cases are possible: $x$ is either above or below $\Gamma$. Consider the first case. Then $d_{\Gamma}(x) \geq 0$ and $B_{d_{\Gamma}(x)}(x)$ touches $\Gamma$ from above. Therefore, $B_{d_{\Gamma}(x)}\left(x+\tau e_{N}\right)$ is above $\Gamma$ and thus, given any $p \in \Gamma$,

$$
\left|p-\left(x+\tau e_{N}\right)\right| \geq d_{\Gamma}(x) .
$$

This proves the desired claim if $x$ is above $\Gamma$.

If $x$ is below $\Gamma$ we distinguish two subcases. If $x+\tau e_{N}$ is above $\Gamma$, then

$$
d_{\Gamma}(x+\tau) \geq 0 \geq d_{\Gamma}(x)
$$

and we are done. If, on the contrary, both $x$ and $x+\tau e_{N}$ are below $\Gamma$, we consider the reflection

$$
\mathcal{R}(y)=\mathcal{R}\left(y^{\prime}, y_{N}\right):=\left(y^{\prime},-y_{N}\right) \quad \forall y \in \mathbb{R}^{N}
$$

and we define $\hat{x}:=\mathcal{R}\left(x+\tau e_{N}\right)$ and $\hat{\Gamma}:=\mathcal{R}(\Gamma)$. Then $\hat{x}$ is above $\hat{\Gamma}$. Since the claim was proved in the first case, we know that $d_{\hat{\Gamma}}\left(\hat{x}+\tau e_{N}\right) \geq d_{\hat{\Gamma}}(\hat{x})$. Hence,

$$
\begin{aligned}
d_{\Gamma}(x) & =-d_{\mathcal{R}(\Gamma)}(\mathcal{R}(x))=-d_{\hat{\Gamma}}\left(\hat{x}+\tau e_{N}\right) \\
& \leq-d_{\hat{\Gamma}}(\hat{x})=-d_{\mathcal{R}(\Gamma)}\left(\mathcal{R}\left(x+\tau e_{N}\right)\right)=d_{\Gamma}\left(x+\tau e_{N}\right) .
\end{aligned}
$$

7 Of course, "above" here is with respect to the $e_{N}$-direction. 


\section{Useful barriers}

The core of the proof of our results begins here. Before going into the details of the arguments, which will be quite technical, we would like to point out some heuristic ideas underlying the construction given below. Roughly, the crucial idea, which goes back to De Giorgi, is that one-dimensional solutions are the ones which encode much information on the system. Following this belief, we will construct two barriers, which are a suitable modification of one-dimensional solutions. The first barrier, built in Lemma 5.1 is radially symmetric. More precisely, it is flat in a ball and then radially increasing. Clearly, since the solution we consider does not possess such symmetry, this barrier may provide good bounds in some direction, but poor bounds in another direction. Therefore, in $\$ 6$. we will have to slide this barrier to obtain information in all the domain we are interested in. In a very non-rigorous way, we may think that the radial growth of this barrier will provide information on "how the solution grows from -1 to +1 ", while the flat part of the barrier controls the "directions along which the level sets of the solution lie". The second barrier we need is constructed in Lemma 5.2. This is a modification of a one-dimensional solution which takes into account the distance from the level sets. Equation (1.5) will then relate second derivatives of this barrier with the mean curvature of the level sets of our rescaled solution, leading to the estimates we need.

We now construct the first comparison function that will be used in the proof of the main result. The function needed to construct such barrier is sketched in the picture.

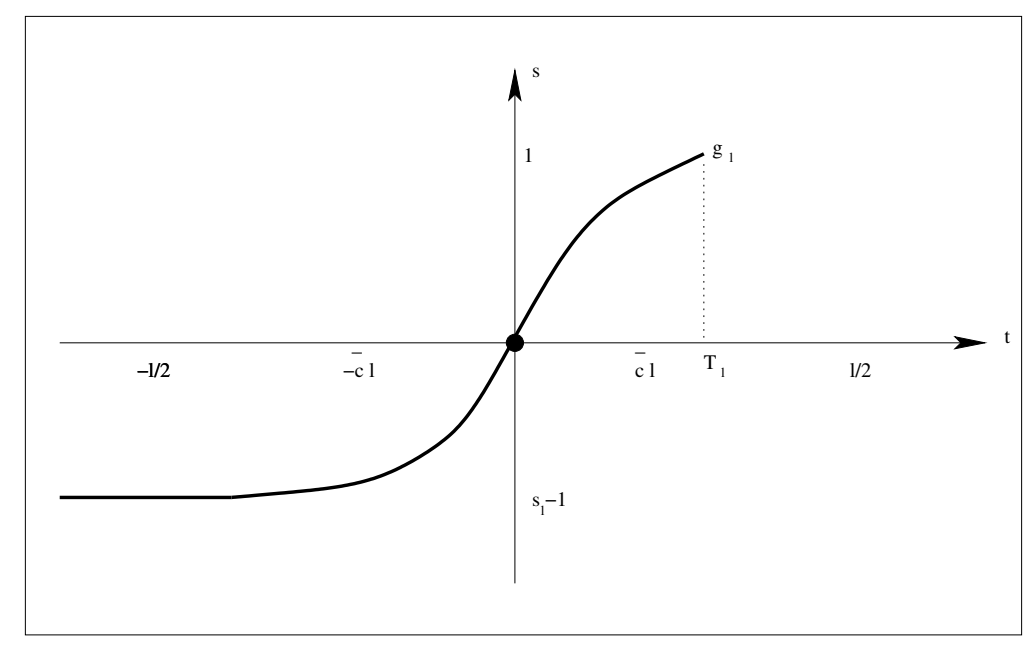

The function $g_{l}$ introduced in Lemma 5.1

Lemma 5.1. There exist universal constants $\bar{l}>1$ and $0<\bar{c} \leq 1 / 2$ so that, if $l \geq \bar{l}$, we can find $T_{l} \in[\bar{c} l, l / 2]$ and a non-decreasing function

$$
g_{l} \in C^{0}\left(-\infty, T_{l}\right) \cap C^{1,1}(-\infty, 0) \cap C^{2}\left(\left(-\bar{c} l, T_{l}\right) \backslash\{0\}\right)
$$


which is constant in an interval I containing $(-\infty,-l / 2]$, with $g_{l}^{\prime}>0$ outside I, satisfies $g_{l}(0)=0, g_{l}\left(T_{l}\right)=1$, and if we define

$$
\Psi^{y, l}(x):=g_{l}(|x-y|-l),
$$

then $\Psi^{y, l}(x)$ is a strict supersolution of 1.5 in the viscosity sense in $B_{T_{l}+l}(y) \backslash \partial B_{l}(y)$.

More precisely, $g_{l}$ is constructed as follows. There exist constants $0<c_{1}<\bar{C}_{1}, \bar{C}_{2}$ so that, if we define

$$
\begin{aligned}
s_{l} & :=e^{-\bar{c}_{1} l}, \\
h_{l}(s) & := \begin{cases}h_{0}(s)-h_{0}\left(s_{l}-1\right)-\frac{\bar{C}_{2}}{l}\left((1+s)^{p}-s_{l}^{p}\right) & \text { if } s_{l}-1<s<0, \\
h_{0}(s)+h_{0}\left(1-s_{l}\right)+\frac{\bar{C}_{2}}{l}\left((1-s)^{p}+s_{l}^{p-1}(1-s)\right) & \text { if } 0 \leq s<1,\end{cases} \\
H_{l}(s) & :=\int_{0}^{s} \frac{(p-1)^{1 / p}}{\left(p h_{l}(\zeta)\right)^{1 / p} d \zeta,} \\
H_{0}(s) & :=\int_{0}^{s} \frac{(p-1)^{1 / p}}{\left(p h_{0}(\zeta)\right)^{1 / p}} d \zeta, \quad \text { for any } s \in(-1,1),
\end{aligned}
$$

then the following holds:

(i) $h_{l}(s)>0$ in $s_{l}-1<s<1$; in particular, $H_{l}$ is well defined and strictly increasing for $s_{l}-1<s<1$ and thus we may define $g_{l}(t):=H_{l}^{-1}(t)$ for $t \in H_{l}\left(s_{l}-1,1\right)$;

(ii) $g_{l}(t)$ is defined to be constantly equal to $s_{l}-1$ for $t \leq H_{l}\left(s_{l}-1\right)$;

(iii) the following estimates on $H_{l}$ hold:

$$
\begin{gathered}
H_{l}(1) \leq l / 2 ; \\
H_{l}\left(s_{l}-1\right) \geq-l / 2 ; \\
\bar{C}_{1}(s) \leq H_{l}(s)-\frac{\frac{1}{l} \log (1-|s|) \quad \forall|s|<1-e^{-\bar{c}_{1} l / 2} ;}{H_{l}\left(1-e^{-\bar{c}_{1} l / 2}\right) \geq \bar{c} l ;} \\
H_{l}\left(e^{-\bar{c}_{1} l / 2}-1\right) \leq-\bar{c} l .
\end{gathered}
$$

Proof. The idea of the proof is that, once $H_{l}$ is well defined, estimates $(5.2)-(5.4)$ and the viscosity supersolution property for $\Psi^{y, l}$ are the core of the matter. Indeed, $\sqrt{5.3}$ says, in particular, that, by construction, $g_{l}$ is constant in $(-\infty,-l / 2]$. Also, estimates [5.4) and (1.2) imply that

$$
H_{l}\left(1-e^{-\bar{c}_{1} l / 2}\right) \geq \mathrm{const} \int_{0}^{1-e^{-\bar{c}_{1} l / 2}} \frac{d \zeta}{1-\zeta}-\text { const }=\text { const } l-1 \geq \bar{c} l,
$$

provided $l$ is large enough and $\bar{c}$ small enough, and, analogously,

$$
H_{l}\left(e^{-\bar{c}_{1} l / 2}-1\right) \leq-\bar{c} l,
$$


proving (5.5) and 5.6). These estimates also imply that $g_{l}$ is strictly increasing at least in $(-\bar{c} l, \bar{c} l)$.

Also, if $T_{l}:=H_{l}(1)$, by $(5.2)$ and $(5.5)$, we have $T_{l} \in[\bar{c} l, l / 2]$. Some careful computation will be needed to show $\Psi^{y, l}$ to be a strict viscosity supersolution of 1.5 at any point where it is defined, except possibly on the sphere $\{|x-y|=l\}$ (the fact that $h_{l}$ may be discontinuous at 0 makes $g_{l}$ not necessarily smooth at 0 and deprives us of information on the value of $\Delta_{p} \Psi^{y, l}$ on the aforesaid sphere). We also remark that the extension in (ii) is $C^{1,1}$, since, by Lemma 4.6 , if $t=H_{l}\left(s_{l}-1\right)$,

$$
g_{l}^{\prime}(t)=\left(\frac{p}{p-1} h_{l}\left(g_{l}(t)\right)\right)^{1 / p}=\left(\frac{p}{p-1} h_{l}\left(s_{l}-1\right)\right)^{1 / p}=0 .
$$

We now deal with the actual proof of Lemma 5.1 in light of the arguments above, we will focus on proving $5.2-5.4$ and the viscosity supersolution property for $\Psi^{y, l}$.

The proof will consider separately the cases $s_{l}-1<s<0$ and $0 \leq s<1$. Let us first consider the case $s_{l}-1 \leq s<0$. From (1.4),

$$
h_{l}(s) \geq h_{0}\left(\theta^{*}-1\right) / 2
$$

if $\theta^{*}-1 \leq s<0$, provided $l$ is suitably large. Also, in light of Lemma 4.2 , we get

$$
h_{0}(s)-h_{0}\left(s_{l}-1\right) \geq c\left((1+s)^{p}-s_{l}^{p}\right),
$$

for $s_{l}-1<s<\theta^{*}-1$, therefore

$$
\text { const }\left(h_{0}(s)-h_{0}\left(s_{l}-1\right)\right) \leq h_{l}(s)
$$

if $s_{l}-1<s<\theta^{*}-1$, provided $l$ is sufficiently large. This, (1.4) and (5.7) say that $h_{l}(s)>0$ in $s_{l}-1<s<0$, showing that $H_{l}$ is well defined and strictly increasing in this case. Also, from the definition of $H_{l}$ and 5.7)-5.9] we gather

$$
\begin{aligned}
-H_{l}\left(s_{l}-1\right) & =\mathrm{const} \int_{s_{l}-1}^{0} \frac{d \zeta}{\left(h_{l}(\zeta)\right)^{1 / p}} \\
& =\operatorname{const}\left(\int_{\theta^{*}-1}^{0} \frac{d \zeta}{\left(h_{l}(\zeta)\right)^{1 / p}}+\int_{s_{l}-1}^{\theta^{*}-1} \frac{d \zeta}{\left(h_{l}(\zeta)\right)^{1 / p}}\right) \\
& \leq \mathrm{const}\left(1+\int_{s_{l}-1}^{\theta^{*}-1} \frac{d \zeta}{\left(h_{l}(\zeta)\right)^{1 / p}}\right) \\
& \leq \mathrm{const}\left(1+\int_{s_{l}-1}^{\theta^{*}-1} \frac{d \zeta}{\left((1+\zeta)^{p}-s_{l}^{p}\right)^{1 / p}}\right) \\
& \leq \mathrm{const}\left(1+\int_{s_{l}-1}^{0} \frac{d \zeta}{\left((1+\zeta)^{p}-s_{l}^{p}\right)^{1 / p}}\right)
\end{aligned}
$$

hence, from Lemma 4.3 , we get

$$
H_{l}\left(s_{l}-1\right) \geq-l / 2
$$

provided $\bar{c}_{1}$ is suitably small, proving 5.3 . 
We now show that $\Psi^{y, l}$ is a viscosity supersolution of 1.5 when $|x-y|<l$ (i.e., when $s=g_{l}(t)<0$; here and in what follows, we often use the notation $t=|x-y|-l$ and $\left.s=g_{l}(t)=\Psi^{y, l}(x)\right)$.

Of course, if $|x-y|<l / 2$, then $\Psi^{y, l}(x)=s_{l}-1$ by 5.3 and the definition of $g_{l}$, and therefore, by Lemma 4.7

$$
\Delta_{p} \Psi^{y, l}(x) \leq 0<h_{0}^{\prime}\left(s_{l}-1\right)=h_{0}^{\prime}\left(\Psi^{y, l}(x)\right),
$$

showing that the viscosity supersolution property of $\Psi^{y, l}$ holds in $\left\{\Psi^{y, l}=s_{l}-1\right\}$ (and thus, in particular, if $|x-y|<l / 2$ ). Hence, we can now concentrate on the case $l / 2 \leq$ $|x-y|<l$ (and, by 5.10 , we may assume that $\Psi^{y, l}(x)>s_{l}-1$ ). In light of Lemma 4.6.

$$
g_{l}^{\prime}(t)=\left(\frac{p}{p-1} h_{l}(g(t))\right)^{1 / p}, \quad g_{l}^{\prime \prime}(t)=\frac{\left(p h_{l}(g(t))\right)^{(2-p) / p}}{(p-1)^{2 / p}} h_{l}^{\prime}\left(g_{l}(t)\right) .
$$

Thus, by Lemma 4.4 , we have

$$
\begin{aligned}
\Delta_{p}\left(\Psi^{y, l}(x)\right) & =(p-1) g^{\prime \prime}(t)\left(g^{\prime}(t)\right)^{p-2}+\left(g^{\prime}(t)\right)^{p-1} \frac{N-1}{|x-y|} \\
& \leq h_{l}^{\prime}\left(g_{l}(t)\right)+K(N-1)\left(h_{l}\left(g_{l}(t)\right)\right)^{(p-1) / p} \frac{1}{|x-y|} \\
& \leq h_{l}^{\prime}\left(g_{l}(t)\right)+\frac{2 K(N-1)\left(h_{l}\left(g_{l}(t)\right)\right)^{(p-1) / p}}{l}
\end{aligned}
$$

for $|x-y| \geq l / 2$, provided $K>0$ is suitably large.

Hence, by definition of $h_{l}$ we get (using again the notation $s=g_{l}(t)$ )

$$
h_{l}(s) \leq h_{0}(s)-h_{0}\left(s_{l}-1\right) \leq h_{0}(s)
$$

and

$$
h_{l}^{\prime}(s)=h_{0}^{\prime}(s)-\frac{p \bar{C}_{2}}{l}(1+s)^{p-1}
$$

in $s_{l}-1<s<0$, hence

$$
\Delta_{p}\left(\Psi^{y, l}(x)\right)<h_{0}^{\prime}(s)-\frac{p \bar{C}_{2}}{l}(1+s)^{p-1}+\frac{2 K(N-1)}{l}\left(h_{0}(s)\right)^{(p-1) / p}
$$

for $s_{l}-1<s<0$. By 1.2 , we get, for $\bar{C}_{2}$ suitably large,

$$
\frac{p \bar{C}_{2}}{l}(1+s)^{p-1} \geq \frac{2 K(N-1)}{l}\left(h_{0}(s)\right)^{(p-1) / p}
$$

and therefore

$$
\Delta_{p}\left(\Psi^{y, l}(x)\right)<h_{0}^{\prime}\left(\Psi^{y, l}(x)\right)
$$

for $s_{l}-1<g_{l}(t)$ and $|x-y| \geq l / 2$.

Estimates 5.10 and 5.14 show $\Psi^{y, l}$ to be a strict viscosity supersolution of 1.5 at any point $x$ so that $|x-y|<l$. 
Let us now prove 5.4) for $e^{-\bar{c}_{1} l / 2}-1<s \leq 0$. Observe that, by definition of $h_{l}$, recalling (1.2),

$$
\begin{aligned}
h_{0}(s)-h_{l}(s) & \leq h_{0}\left(s_{l}-1\right)+\frac{\bar{C}_{2}}{l}\left((1+s)^{p}-s_{l}^{p}\right) \leq C s_{l}^{p}+\frac{\bar{C}_{2}}{l}\left((1+s)^{p}-s_{l}^{p}\right) \\
& \leq \frac{2 \bar{C}_{2}}{l}(1+s)^{p}
\end{aligned}
$$

provided $l$ is sufficiently large. Furthermore, from (5.7)-(5.9), it follows that

$$
h_{l}(s) \geq \operatorname{const}(1+s)^{p}
$$

if $e^{-\bar{c}_{1} l / 2}-1<s \leq 0$ and $l$ is large enough. Also, using Lemma 4.1. we obtain

$$
\begin{aligned}
H_{0}(s)-H_{l}(s) & =\int_{s}^{0} \frac{1}{\left(\frac{p}{p-1} h_{l}(\zeta)\right)^{1 / p}}-\frac{1}{\left(\frac{p}{p-1} h_{0}(\zeta)\right)^{1 / p}} d \zeta \\
& =\text { const } \int_{s}^{0} \frac{\left(h_{0}(\zeta)\right)^{1 / p}-\left(h_{l}(\zeta)\right)^{1 / p}}{\left(h_{0}(\zeta) h_{l}(\zeta)\right)^{1 / p}} d \zeta \\
& \leq \text { const } \int_{s}^{0} \frac{\left(h_{0}(\zeta)-h_{l}(\zeta)\right) d \zeta}{\left(\left(h_{0}(\zeta)\right)^{(p-1) / p}+\left(h_{l}(\zeta)\right)^{(p-1) / p}\right)\left(h_{0}(\zeta) h_{l}(\zeta)\right)^{1 / p}} \\
& \leq \text { const } \int_{s}^{0} \frac{h_{0}(\zeta)-h_{l}(\zeta)}{h_{0}(\zeta)\left(h_{l}(\zeta)\right)^{1 / p}} d \zeta .
\end{aligned}
$$

Consequently, from (1.2), 5.16) and 5.15,

$$
H_{0}(s)-H_{l}(s) \leq \frac{\text { const }}{l} \int_{s}^{0} \frac{1}{1+\zeta} d \zeta \leq-\frac{\text { const }}{l} \log (1+s),
$$

thus proving 5.4 for $e^{-\bar{c}_{1} l / 2}-1<s \leq 0$. This completes the proof in the case $s_{l}-1<s<0$.

Let us now consider the case $0 \leq s<1$. In this case, $h_{l}>0$ by inspection, thus $H_{l}$ is well defined and strictly increasing in $[0,1)$. Setting $t=|x-y|-l$ and $s=g_{l}(t)=\Psi^{y, l}(x)$, we notice that $s \geq 0$ corresponds to $|x-y| \geq l$, therefore, arguing as in (5.11), we have

$$
\Delta_{p}\left(\Psi^{y, l}(x)\right)<h_{l}^{\prime}(s)+\frac{K(N-1)}{l}\left(h_{l}(s)\right)^{(p-1) / p}
$$

if $|x-y| \geq l$, provided $K$ is large enough. Since, by definition of $h_{l}$ and $(1.2)$,

$$
h_{l}(s) \leq \mathrm{const}\left(h_{0}(s)+h_{0}\left(1-s_{l}\right)\right)
$$

for $\bar{C}_{2}$ large enough, it follows that

$$
\begin{aligned}
\Delta_{p}\left(\Psi^{y, l}(x)\right) & <h_{0}^{\prime}(s)-\frac{\bar{C}_{2}}{l}\left(p(1-s)^{p-1}+s_{l}^{p-1}\right)+\frac{K(N-1)}{l}\left(h_{0}(s)+h_{0}\left(1-s_{l}\right)\right)^{(p-1) / p} \\
& \leq h_{0}^{\prime}(s)
\end{aligned}
$$


if $\bar{C}_{2}$ is suitably large, where, in the last estimate, 1.2 has been used once more together with the simple inequality $(a+b)^{q} \leq 2^{q}\left(a^{q}+b^{q}\right)$ with $q:=(p-1) / p$. Thus $\Psi^{y, l}(x)$ is a strict viscosity supersolution of $(1.5)$ for $|x-y|>l$, provided $\Psi^{y, l}(x)$ is well defined.

We now need to prove 5.4 in the case $0 \leq s<1-e^{-\bar{c}_{1} l / 2}$. To this end, first notice that, if $0 \leq s<1-e^{-\bar{c}_{1} l / 2}$, we have $1-s>\sqrt{s_{l}}$ and therefore

$$
s_{l}^{p} \leq s_{l}^{p / 2}(1-s)^{p} \leq \frac{1}{l}(1-s)^{p}
$$

if $l$ is large enough. The definition of $h_{l},(1.2)$ and 5.18$)$ imply that

$$
h_{l}(s)-h_{0}(s) \leq \text { const } s_{l}^{p}+\frac{\text { const }}{l}(1-s)^{p} \leq \frac{\text { const }}{l}(1-s)^{p},
$$

for $0 \leq s<1-e^{-\bar{c}_{1} l / 2}$. On the other hand, the definition of $h_{l}$ and 1.2 lead to

$$
h_{l}(s) \geq h_{0}(s)+h_{0}\left(1-s_{l}\right) \geq \operatorname{const}(1-s)^{p}
$$

for $0 \leq s<1-e^{-\bar{c}_{1} l / 2}$. Also, from Lemma 4.1 .

$$
\begin{aligned}
H_{0}(s)-H_{l}(s) & =\int_{0}^{s} \frac{1}{\left(\frac{p}{p-1} h_{0}(\zeta)\right)^{1 / p}}-\frac{1}{\left(\frac{p}{p-1} h_{l}(\zeta)\right)^{1 / p}} d \zeta \\
& \leq \mathrm{const} \int_{0}^{s} \frac{h_{l}(\zeta)-h_{0}(\zeta)}{\left(\left(h_{l}(\zeta)\right)^{(p-1) / p}+\left(h_{0}(\zeta)\right)^{(p-1) / p}\right)\left(h_{l}(\zeta) h_{0}(\zeta)\right)^{1 / p}} d \zeta \\
& \leq \mathrm{const} \int_{0}^{s} \frac{h_{l}(\zeta)-h_{0}(\zeta)}{h_{0}(\zeta)\left(h_{l}(\zeta)\right)^{1 / p}} d \zeta
\end{aligned}
$$

Then, from 5.19) -5.21) and 1.2,

$$
H_{0}(s)-H_{l}(s) \leq \frac{\text { const }}{l} \int_{0}^{s} \frac{d \zeta}{1-\zeta}=-\frac{\text { const }}{l} \log (1-s)
$$

if $0 \leq s<1-e^{-\bar{c}_{1} l / 2}$. This indeed proves 5.4 in the case $0 \leq s<1-e^{-\bar{c}_{1} l / 2}$.

Let us now prove [5.2]. Using the definitions of $H_{l}, h_{l}$ and (1.2), we get

$$
\begin{aligned}
H_{l}(1) & \leq \text { const } \int_{0}^{1} \frac{d \zeta}{\left(h_{0}(\zeta)+h_{0}\left(1-s_{l}\right)\right)^{1 / p}} \leq \text { const } \int_{0}^{1} \frac{d \zeta}{\left((1-\zeta)^{p}+s_{l}^{p}\right)^{1 / p}} \\
& \leq \text { const } \int_{0}^{1} \frac{d \zeta}{1-\zeta+s_{l}} \leq \text { const } \log \frac{1}{s_{l}} .
\end{aligned}
$$

This proves 5.2 provided $\bar{c}_{1}$ is chosen to be suitably small, and ends the proof of Lemma 5.1

We now introduce an appropriate modification of the barrier in Lemma 5.1] in order to deal with the distance function: 
Lemma 5.2. Let $0<\varepsilon \leq \sigma \leq \delta<1, \xi \in \mathbb{R}^{N-1}$, and $M \in \operatorname{Mat}((N-1) \times(N-1))$. Let $\Gamma$ be the hypersurface defined as

$$
\Gamma:=\left\{x_{N}=\frac{\varepsilon}{2} x^{\prime} \cdot M x^{\prime}+\sigma \xi \cdot x^{\prime}\right\} \cap\left\{\left|x^{\prime}\right|<\sigma / \varepsilon\right\}
$$

and assume that

$$
\operatorname{tr} M \geq \delta, \quad\|M\| \leq 2 / \delta, \quad|\xi| \leq 1 / \delta
$$

Define $d_{\Gamma}(x)$ to be the signed distance from $x$ to $\Gamma$, with the assumption that $d_{\Gamma}$ is positive 8 above $\Gamma$.

Then there exist functions $\sigma_{0}:(0,+\infty) \rightarrow(0,1)$ and $C_{0}:(0,+\infty) \rightarrow[1,+\infty)$ and $T_{\varepsilon, \delta} \in\left[0, C_{0}(\delta) \log (1 / \varepsilon)\right]$ such that, if $\varepsilon \leq \sigma \leq \sigma_{0}(\delta)$, we can find a non-decreasing function $g_{\Gamma} \in C^{1,1}\left(-\infty, T_{\varepsilon, \delta}\right)$, constant in $\left(-\infty,-C_{0}(\delta) \log (1 / \varepsilon)\right]$, such that $g_{\Gamma}(0)=0$, $g_{\Gamma}\left(T_{\varepsilon, \delta}\right)=1, g_{\Gamma}$ is $C^{2}$ with $g_{\Gamma}^{\prime}$ non-vanishing outside the set where $g$ is constant, and for which $g_{\Gamma}\left(d_{\Gamma}(x)\right)$ is a strict viscosity supersolution of $(1.5)$ in its domain of definition (that is, provided $\left.d_{\Gamma}(x)<T_{\varepsilon, \delta}\right)$.

More precisely, $g_{\Gamma}$ is constructed as follows. Let $c_{1}>0$ be suitably small and let $\rho \in C^{1}(\mathbb{R})$ be a non-decreasing function so that $\rho(0)=0, \rho(s)=-1$ for $s \leq-1 / 2$ and $\rho(s)=1$ for $s \geq 1 / 2$. For any $s \in(-1,1)$, define

$$
h_{\Gamma}(s):=\max \left\{0, h_{0}(s)+c_{1} \delta \varepsilon \rho(s)\right\} .
$$

Let $s_{\delta, \varepsilon}$ be the point near -1 for which $h_{0}\left(s_{\delta, \varepsilon}\right)=c_{1} \delta \varepsilon$. Define also

$$
H_{\Gamma}(s):=\int_{0}^{s} \frac{(p-1)^{1 / p} d \zeta}{\left(p h_{\Gamma}(\zeta)\right)^{1 / p}}
$$

Then:

(i) There exists a constant $c^{\sharp} \in(0,1)$ so that

$$
c^{\sharp}(\delta \varepsilon)^{1 / p} \leq 1+s_{\delta, \varepsilon} \leq \frac{1}{c^{\sharp}}(\delta \varepsilon)^{1 / p} ;
$$

(ii) for any $s_{\delta, \varepsilon}<s \leq 1$,

$$
h_{\Gamma}(s)>0
$$

in particular, $H_{\Gamma}$ is well defined and strictly increasing in $\left[s_{\delta, \varepsilon}, 1\right]$ and thus we may define $g_{\Gamma}(t):=H_{\Gamma}^{-1}(t)$ for any $t \in\left[H_{\Gamma}\left(s_{\delta, \varepsilon}\right), H_{\Gamma}(1)\right]$ and extend $g_{\Gamma}(t)$ to be constantly $s_{\delta, \varepsilon}$ for $t \leq H_{\Gamma}\left(s_{\delta, \varepsilon}\right)$. In particular, if $g_{\Gamma}(t)>s_{\delta, \varepsilon}$, then $g_{\Gamma}^{\prime}(t)>0$.

8 Again, "above" is with respect to the $e_{N}$-direction. For some properties of the distance function, see [9]. 
Proof. First observe that (5.23) follows from (1.2): indeed, if $c$ and $C$ are as in (1.2), then

$$
\left(\frac{c_{1}}{C}\right)^{1 / p}(\delta \varepsilon)^{1 / p} \leq 1+s_{\delta, \varepsilon} \leq\left(\frac{c_{1}}{c}\right)^{1 / p}(\delta \varepsilon)^{1 / p} .
$$

Also, with no loss of generality, we may assume $s_{\delta, \varepsilon}<-1+\theta^{*}$, in order to use (1.3). Note that since by $(1.3), h_{0}$ is increasing in $\left[s_{\delta, \varepsilon}, \theta^{*}\right)$, we get $h_{0}\left(s_{\delta, \varepsilon}\right)>c_{1} \delta \varepsilon$ in $\left(s_{\delta, \varepsilon}, \theta^{*}\right)$. Moreover, from (1.2), if $c_{1}$ is small enough, we may suppose $h_{0}(s)>c_{1} \delta \varepsilon$ for $s_{\delta, \varepsilon}<s<0$. From the above discussions, (5.24) follows.

Notice that the constant extension of $g_{\Gamma}$ is $C^{1,1}$ since, by Lemma 4.6 if $t=H_{\Gamma}\left(s_{\delta, \varepsilon}\right)$,

$$
g_{\Gamma}^{\prime}(t)=\left(\frac{p}{p-1} h_{\Gamma}\left(g_{\Gamma}(t)\right)\right)^{1 / p}=\left(\frac{p}{p-1} h_{\Gamma}\left(s_{\delta, \varepsilon}\right)\right)^{1 / p}=0 .
$$

To estimate the domain on which $g_{\Gamma}$ is strictly increasing we have therefore to estimate $H_{\Gamma}\left(s_{\delta, \varepsilon}\right)$ and $H_{\Gamma}(1)$. Using Lemma 4.2 , one obtains

$$
h_{\Gamma}(s) \geq h_{0}(s)-c_{1} \delta \varepsilon=h_{0}(s)-h_{0}\left(s_{\delta, \varepsilon}\right) \geq \operatorname{const}\left((1+s)^{p}-\left(1+s_{\delta, \varepsilon}\right)^{p}\right)^{1 / p}
$$

for any $s \in\left[s_{\delta, \varepsilon},-1+\theta^{*}\right]$. On the other hand, for any $\left.s \in\left[-1+\theta^{*}, 0\right], 11.4\right]$ implies that

$$
h_{\Gamma}(s) \geq h_{0}\left(-1+\theta^{*}\right)-c_{1} \delta \varepsilon \geq h_{0}\left(-1+\theta^{*}\right) / 2 .
$$

Therefore, using the definition of $H_{\Gamma}, 5.23$, 5.25) and 5.26, and making use of Lemma 4.3 , we get

$$
\begin{aligned}
-H_{\Gamma}\left(s_{\delta, \varepsilon}\right) & =\int_{s_{\delta, \varepsilon}}^{0} \frac{(p-1)^{1 / p} d \zeta}{\left(p h_{\Gamma}(\zeta)\right)^{1 / p}}=\int_{-1+\theta^{*}}^{0} \frac{(p-1)^{1 / p} d \zeta}{\left(p h_{\Gamma}(\zeta)\right)^{1 / p}}+\int_{s_{\delta, \varepsilon}}^{-1+\theta^{*}} \frac{(p-1)^{1 / p} d \zeta}{\left(p h_{\Gamma}(\zeta)\right)^{1 / p}} \\
& \leq \operatorname{const}\left(1+\int_{s_{\delta, \varepsilon}}^{-1+\theta^{*}} \frac{d \zeta}{\left((1+\zeta)^{p}-\left(1+s_{\delta, \varepsilon}\right)^{p}\right)^{1 / p}}\right) \leq C_{0}(\delta) \log \frac{1}{\varepsilon},
\end{aligned}
$$

or, equivalently,

$$
H_{\Gamma}\left(s_{\delta, \varepsilon}\right) \geq C_{0}(\delta) \log \varepsilon .
$$

This completes the desired estimate on $H_{\Gamma}\left(s_{\delta, \varepsilon}\right)$.

Let us now estimate $H_{\Gamma}(1)$ : from the definition of $h_{\Gamma}$ and (1.2),

$$
\begin{aligned}
H_{\Gamma}(1) & =\int_{0}^{1} \frac{(p-1)^{1 / p} d \zeta}{\left(p h_{\Gamma}(\zeta)\right)^{1 / p}} \leq \int_{0}^{1 / 2} \frac{\operatorname{const} d \zeta}{1-\zeta}+\int_{1 / 2}^{1} \frac{\text { const } d \zeta}{\left(c(1-\zeta)^{p}+c_{1} \delta \varepsilon\right)^{1 / p}} \\
& \leq \int_{0}^{1 / 2} \frac{\text { const } d \zeta}{1-\zeta}+\int_{1 / 2}^{1} \frac{\operatorname{const} d \zeta}{1-\zeta+(\delta \varepsilon)^{1 / p}} \\
& \leq \operatorname{const}(1-\log (\delta \varepsilon)) \leq-C_{0}(\delta) \log \varepsilon
\end{aligned}
$$

The claims on the domain of $g_{\Gamma}$ are thus consequences of 5.27) and 5.28). 
Now we deal with the proof of the viscosity supersolution property of $g_{\Gamma}$. First of all, notice that in an appropriate coordinate system we have

$$
D^{2} d_{\Gamma}=\operatorname{diag}\left(\frac{-k_{1}}{1-d_{\Gamma} k_{1}}, \ldots, \frac{-k_{N-1}}{1-d_{\Gamma} k_{N-1}}, 0\right) \in \operatorname{Mat}(N \times N),
$$

where the $k_{i}$ 's are the principal curvatures of $\Gamma$ at the point where the distance is realized (see $\$ 14.6$ in [9] for further details). We also define $P$ as the paraboloid describing $\Gamma$, i.e.,

$$
P\left(x^{\prime}\right):=\frac{\varepsilon}{2} x^{\prime} \cdot M x^{\prime}+\sigma \xi \cdot x^{\prime}
$$

Notice that, by construction, $|\nabla P| \leq 1$; thus, by the mean curvature equation (see, for instance, equation (14.103) of [9]), it follows that

$$
\begin{aligned}
\sum_{i=1}^{N-1} k_{i} & =\sum_{1 \leq i \leq N-1} \partial_{i}\left(\frac{\partial_{i} P}{\sqrt{1+|\nabla P|^{2}}}\right)=\frac{\Delta P}{\sqrt{1+|\nabla P|^{2}}}-\frac{\left(D^{2} P \nabla P\right) \cdot \nabla P}{\left(1+|\nabla P|^{2}\right)^{3 / 2}} \\
& \geq \frac{1}{2} \Delta P-\text { const }|\nabla P|^{2}\left\|D^{2} P\right\| .
\end{aligned}
$$

Consequently, if $x$ is so that $\left|d_{\Gamma}(x)\right| \leq C_{0}(\delta) \log (1 / \varepsilon)$, since $\left|k_{i}\right| \leq C_{1}(\delta) \varepsilon$, we have

$$
\begin{aligned}
\Delta d_{\Gamma} & \leq \sum_{i=1}^{N-1} \frac{-k_{i}}{1-d_{\Gamma} k_{i}} \leq-\sum_{i=1}^{N-1} k_{i}+2\left(C_{1}(\delta) \varepsilon\right)^{2} \log \frac{1}{\varepsilon} \\
& \leq-\frac{1}{2} \Delta P+\mathrm{const}|\nabla P|^{2}\left\|D^{2} P\right\|+C_{1}(\delta) \varepsilon^{3 / 2} \\
& \leq-\frac{\varepsilon \delta}{2}+C_{2}(\delta)\left(\varepsilon \sigma^{2}+\varepsilon^{3 / 2}\right) \leq-\frac{\varepsilon \delta}{2}+C_{3}(\delta) \varepsilon \sigma^{1 / 2}
\end{aligned}
$$

Therefore, if $d_{\Gamma}(x) \in\left(H_{\Gamma}\left(s_{\delta, \varepsilon}\right), H_{\Gamma}(1)\right)$ (and thus, by 5.27) and 5.28), $\left|d_{\Gamma}(x)\right| \leq$ $C_{0}(\delta) \log (1 / \varepsilon)$ and $\left.g_{\Gamma}^{\prime}\left(d_{\Gamma}(x)\right)>0\right)$, by Lemma 4.5 we have

$$
\begin{aligned}
\Delta_{p}\left(g_{\Gamma}(t)\right) & =(p-1)\left(g_{\Gamma}^{\prime}(t)\right)^{p-2} g_{\Gamma}^{\prime \prime}(t)+\left(g_{\Gamma}^{\prime}(t)\right)^{p-1} \Delta d_{\Gamma}(t) \\
& \leq(p-1)\left(g_{\Gamma}^{\prime}(t)\right)^{p-2} g_{\Gamma}^{\prime \prime}(t)-\frac{\varepsilon}{2}\left(\delta-C_{4}(\delta) \sigma^{1 / 2}\right)\left(g_{\Gamma}^{\prime}(t)\right)^{p-1}
\end{aligned}
$$

where we are using the notation $t=d_{\Gamma}(x)$. Taking into account Lemma 4.6, by 5.30 we get

$$
\Delta_{p}\left(g_{\Gamma}(t)\right) \leq h_{\Gamma}^{\prime}(s)-\frac{\varepsilon}{2}\left(\delta-C_{4}(\delta) \sigma^{1 / 2}\right)\left(\frac{p}{p-1} h_{\Gamma}(s)\right)^{(p-1) / p}
$$

where we are using the notation $s=g_{\Gamma}\left(d_{\Gamma}(x)\right)$.

Now we choose $\sigma_{0}(\delta)$ small such that $\delta-C_{4}(\delta) \sigma^{1 / 2} \geq \delta / 2$ for $\sigma \leq \sigma_{0}(\delta)$. Thus, if $\left|d_{\Gamma}(x)\right| \leq C_{0}(\delta) \log (1 / \varepsilon)$ (and so $\left.s=g_{\Gamma}\left(d_{\Gamma}(x)\right)>s_{\delta, \varepsilon}\right)$, we gather (recall also (5.24)) that

$$
\begin{aligned}
\Delta_{p}\left(g_{\Gamma}(t)\right) & \leq h_{\Gamma}^{\prime}(s)-\text { const } \delta \varepsilon\left(h_{\Gamma}(s)\right)^{(p-1) / p} \\
& \leq h_{0}^{\prime}(s)+c_{1} \delta \varepsilon \rho^{\prime}(s)-\text { const } \delta \varepsilon\left(h_{0}(s)+c_{1} \delta \varepsilon \rho(s)\right)^{(p-1) / p} .
\end{aligned}
$$


We now claim that

$$
c_{1} \rho^{\prime}(s)-\text { const }\left(h_{0}(s)+c_{1} \delta \varepsilon \rho(s)\right)^{(p-1) / p}<0
$$

for any $s \in\left(s_{\delta, \varepsilon}, 1\right)$, provided $c_{1}$ is small enough. Indeed, if $s \leq-1 / 2$ or $s \geq 1 / 2$, then $\rho^{\prime}(s)=0$ and therefore the left hand side of 5.32 is under control. On the other hand, if $s \in(-1 / 2,1 / 2)$, then setting $c^{*}:=\inf _{s \in[-1 / 2,1 / 2]} h_{0}(s)$ (which is strictly positive on account of (1.2), we bound the left hand side of (5.32) by

$$
c_{1}\left\|\rho^{\prime}\right\|_{\infty}-\operatorname{const}\left(c^{*}\right)^{(p-1) / p},
$$

which is negative for suitably small $c_{1}$. This proves 5.32 .

Therefore, by virtue of (5.31) and 5.32), if $d_{\Gamma}(x) \in\left(H_{\Gamma}\left(s_{\delta, \varepsilon}\right), H_{\Gamma}(1)\right)$, we get

$$
\Delta_{p}\left(g_{\Gamma}(t)\right)<h_{0}^{\prime}\left(g_{\Gamma}(t)\right) .
$$

If else $d_{\Gamma}(x) \leq H_{\Gamma}\left(s_{\delta, \varepsilon}\right)$, we have

$$
\Delta_{p}\left(g_{\Gamma}(t)\right)=0<h_{0}^{\prime}\left(s_{\delta, \varepsilon}\right)=h_{0}^{\prime}\left(g_{\Gamma}(t)\right),
$$

thanks to Lemma 4.7

\section{Sliding methods}

We now use the barriers introduced in $\$ 5$ and an appropriate sliding technique to deduce an estimate on the curvature of touching paraboloids for solutions of 1.5 . Roughly speaking, the barriers $\Psi^{y, l}$ defined above provide a good constraint for touching points, since the latter can lie only in the set where the barriers fail to be supersolutions (that is, on their zero level set). More precisely, the following results hold:

Lemma 6.1. Let $u$ be a weak Sobolev subsolution of $(1.5)$. Then $u$ and $\Psi^{y, l}$ cannot coincide in any open domain.

Proof. For short, let $\Psi:=\Psi^{y, l}, B:=B_{T_{l}+l}(y), B^{\prime}:=B_{l}(y)$. Furthermore, let $\tau(l) \in$ $[l / 2,(1-\bar{c}) l]$ be so that $\Psi$ is flat in $B^{\prime \prime}:=B_{\tau(l)}(y)$. Then $B^{\prime \prime} \subset B^{\prime} \subset B$, the domain of definition of $\Psi$ is $B$, and $\Psi$ is $C^{2}$ outside $\partial B^{\prime} \cup \partial B^{\prime \prime}$. Suppose by contradiction that $u=\Psi$ in some ball $\mathfrak{B} \subseteq B$. Possibly taking a smaller ball, we may and do assume that

$$
\mathfrak{B} \subset(\Omega \cap B) \backslash\left(\partial B^{\prime} \cup \partial B^{\prime \prime}\right),
$$

hence $u=\Psi$ is $C^{2}$ in $\mathfrak{B}$; therefore, for any non-negative smooth function $\varphi$ supported in $\mathfrak{B}$, we deduce from 1.5 that

$$
\begin{aligned}
\int h_{0}(\Psi) \varphi & >-\int|\nabla \Psi|^{p-2} \nabla \Psi \cdot \nabla \varphi=-\int|\nabla u|^{p-2} \nabla u \cdot \nabla \varphi \geq \int h_{0}(u) \varphi \\
& =\int h_{0}(\Psi) \varphi,
\end{aligned}
$$

which is obviously impossible. 
Lemma 6.2. Fix $y \in \mathbb{R}^{N}$ and let $l>0$ be suitably large. Let $u$ be a weak Sobolev subsolution of 1.5 in some domain $\Omega$. Suppose that $u \in C^{1}(\Omega)$ and 9 that $|u| \leq 1$. Suppose that $\Psi^{y, l}$ touches $u$ from above at $x^{\star}$, that is, $\Psi^{y, l} \geq u$ in their common domain of definition $\Omega \cap B_{T_{l}+l}(y)$ and $\Psi^{y, l}\left(x^{\star}\right) \geq u\left(x^{\star}\right)$, with $x^{\star}$ in the closure of $\Omega \cap B_{T_{l}+l}(y)$. Then either $x^{\star} \in \partial \Omega$ or $u\left(x^{\star}\right)=\Psi^{y, l}\left(x^{\star}\right)=0$.

Proof. For short, we write $\Psi:=\Psi^{y, l}, B:=B_{T_{l}+l}(y), B^{\prime}:=B_{l}(y)$. Assume that

$$
x^{\star} \notin \partial \Omega
$$

we will show that then $\Psi\left(x^{\star}\right)=0$. For this, first we prove that

$$
x^{\star} \notin \partial B .
$$

Indeed, suppose the contrary. Let us consider the "radial direction"

$$
w:=\frac{x^{\star}-y}{\left|x^{\star}-y\right|} .
$$

Then, by the construction in Lemma 5.1, $\Psi\left(x^{\star}\right)=1$ and $\nabla \Psi\left(x^{\star}\right) \cdot w>0$. On the other hand, $u \leq 1$ and, since $u\left(x^{\star}\right)=1$, we have $\nabla u\left(x^{\star}\right)=0$ (thanks to (6.1) and the assumption that $\left.u \in C^{1}(\Omega)\right)$. Let $\hat{u}:=u-\Psi$. From the above discussion, $\hat{u} \leq 0$ in $B \cap \Omega$ and $\hat{u}\left(x^{\star}\right)=0$, therefore

$$
\nabla \hat{u}\left(x^{\star}\right) \cdot w \geq 0
$$

But then

$$
0 \leq \nabla(u-\Psi)\left(x^{\star}\right) \cdot w=-\nabla \Psi\left(x^{\star}\right) \cdot w<0,
$$

which is a contradiction. This proves 6.2.

Due to 6.1 and 6.2,

$$
x^{\star} \text { is in the interior of } \Omega \cap B .
$$

Moreover, by construction, $\Psi$ has no points with vanishing gradient outside the region where it is flat: more precisely, $\Psi$ is flat in $B^{\prime \prime}:=B_{\tau(l)}(y)$ for some $\tau(l) \in[l / 2,(1-\bar{c}) l]$, $B^{\prime \prime} \subset B^{\prime} \subset B, \Psi$ is constantly equal to $-1+e^{- \text {const } l}$ in $B^{\prime \prime}$, and if

$$
Z_{u, \Psi}:=\{|\nabla u|+|\nabla \Psi|=0\},
$$

then

Also, by Lemma 6.1 .

$$
Z_{u, \Psi} \subseteq\{|\nabla \Psi|=0\} \subseteq \overline{B^{\prime \prime}}
$$

$u$ and $\Psi$ cannot agree in any open domain.

Then, by 6.4, 6.5 and Corollary 3.3 (and recalling 6.3) $), x^{\star}$ may only lie either on $\partial B^{\prime}$ (where $\Psi=0$ and it fails to be a supersolution) or in the closure of $B^{\prime \prime}$.

9 The assumptions on the subsolution $u$ in Lemma 6.2 (and in the other analogous results of this section) are, in particular, fulfilled in case $u$ is a weak Sobolev solution of 1.5 satisfying $|u| \leq 1$, since the $C^{1}$-regularity is given by the results in [8] or [20]. 
Thus, Lemma 6.2 is proved once we show that

$$
x^{\star} \text { does not lie in the closure of } B^{\prime \prime} \text {. }
$$

The proof of (6.6) is, once more, by contradiction. If (6.6) were false, then $(6.5)$ implies that we have $u<\Psi$ in the interior of a suitable ball $\hat{B} \subseteq B^{\prime \prime}$ and $u\left(y^{\prime}\right)=\Psi\left(y^{\prime}\right)$ for some $y^{\prime} \in \partial \hat{B}$. Since $\nabla \Psi^{t}\left(y^{\prime}\right)=0$ and $u \in C^{1}$ by assumption, we have

$$
\nabla u\left(y^{\prime}\right)=0 .
$$

Set $u^{\star}:=-u+1-e^{- \text {const } l}$. Then, by 1.3 , if $l$ is sufficiently large, we have, in a weak sense,

$$
-\Delta_{p} u^{\star}=\Delta_{p} u \geq h_{0}^{\prime}(u) \geq \text { const }(1+u)^{p-1} \geq 0
$$

in $\hat{B}$; also, $u^{\star}>0$ in the interior of $\hat{B}$ and $u^{\star}\left(y^{\prime}\right)=0$; consequently, by Theorem 3.4 (with $c=0$ and $g=0$ ) it would follow that $\nabla u^{\star}\left(y^{\prime}\right) \neq 0$ and so $\nabla u\left(y^{\prime}\right) \neq 0$, contradicting 6.7 .

This proves 6.6 and thus completes the proof of Lemma 6.2.

Here is another result which allows us to bound subsolutions of 1.5 by the barriers $\Psi^{y, l}$ :

Proposition 6.3. Let $u$ be a weak subsolution of $(1.5)$ in the domain $\Omega \subseteq \mathbb{R}^{N}$, with $|u(x)| \leq 1$ for any $x \in \Omega$. Assume that $u \in C^{1}(\Omega)$. Let $y \in \mathbb{R}^{N}$ and $l>0$ be such that

$$
B_{l+T_{l}}(y) \subset\left\{x \in \Omega: u(x) \leq-1+\theta^{*}\right\} .
$$

Then

$$
u(x) \leq \Psi^{y, l}(x)
$$

for any $x \in B_{l+T_{l}}(y)$, provided $l$ is sufficiently large.

Proof. Notice that $\Psi^{y, l}$ is defined on $B_{l+T_{l}}(y)$ and that, if $x \in B_{l+T_{l}}(y) \backslash B_{l}(y)$, then

$$
\Psi^{y, l}(x) \geq 0>-1+\theta^{*} \geq u(x) .
$$

Therefore, due to 6.8, if the claim of Proposition 6.3 were false, there would be an open set $\mathfrak{U}$ such that

$$
\mathfrak{U} \subset B_{l}(y) \subset \Omega \cap\left\{u<-1+\theta^{*}\right\},
$$

so that $\Psi^{y, l}<u$ in $\mathfrak{U}$, and $\Psi^{y, l}=u$ on $\partial \mathfrak{U}$. Consequently, there exists $\kappa>0$ so that $v:=$ $u-\kappa \leq \Psi^{y, l}$ in $\mathfrak{U}, v<\Psi^{y, l}$ in $\partial \mathfrak{U}$ and $v\left(x^{\star}\right)=\Psi\left(x^{\star}\right)$ for some $x^{\star} \in \mathfrak{U}$. Note also that

$$
v\left(x^{\star}\right)=\Psi\left(x^{\star}\right) \in(-1,0),
$$

since $x^{\star} \in \mathfrak{U} \subset B_{l}(y)$, and therefore

$$
x^{\star} \in \mathfrak{V}:=\mathfrak{U} \cap\{|v|<1\} .
$$

Since $h_{0}^{\prime}$ is increasing in $B_{l+T_{l}}(y)$ (thanks to 6.8 and our assumption on page 320 ,

$$
\Delta_{p} v=\Delta_{p} u \geq h_{0}^{\prime}(u)=h_{0}^{\prime}(v+\kappa) \geq h_{0}^{\prime}(v)
$$

weakly in $\mathfrak{V}$. Consequently, from Lemma 6.2 we deduce that either $x^{\star} \in \partial \mathfrak{V}$ or $v\left(x^{\star}\right)=0$. The first assertion would contradict (6.11), while the second one is ruled out by 6.10). This provides the contradiction which proves the desired result. 
Proposition 6.3 can be easily sharpened, giving a strict inequality, in the following way:

Corollary 6.4. Let $u$ be a weak subsolution of 1.5 in the domain $\Omega \subseteq \mathbb{R}^{N}$, with $|u(x)| \leq 1$ for any $x \in \Omega$. Assume that $u \in C^{1}(\Omega)$. Let $y \in \mathbb{R}^{N}$ and $l>0$ be such that

$$
B_{l+T_{l}}(y) \subset\left\{x \in \Omega: u(x) \leq-1+\theta^{*}\right\} .
$$

Then

$$
u(x)<\Psi^{y, l}(x)
$$

for any $x \in B_{l+T_{l}}(y)$, provided $l$ is sufficiently large.

Proof. By Proposition 6.3. we know that $u \leq \Psi^{y, l}$. If there existed $x^{\star}$ for which equality holds, then 6.13 and Lemma 6.2 would imply that $u\left(x^{\star}\right)=\Psi^{y, l}\left(x^{\star}\right)=0$, in contradiction with 6.13).

A result analogous to Lemma 6.2 holds for the barrier $g_{\Gamma}\left(d_{\Gamma}\right)$ constructed in Lemma 5.2 For the convenience of the reader we will now provide some details on this:

Lemma 6.5. Let $u$ be a weak Sobolev subsolution of (1.5) in some domain $\Omega$. Suppose that $u \in C^{1}(\Omega)$ and that $|u| \leq 1$. Suppose that $g_{\Gamma}\left(d_{\Gamma}\right)$ touches $u$ from above at $x^{\star}$. Then $x^{\star} \in \partial \Omega$.

Proof. First notice that $u$ and $g_{\Gamma}\left(d_{\Gamma}\right)$ cannot be identically equal in any open set: this can be proven by an easy modification of the argument in Lemma 6.1. By Corollary 3.3. we infer that interior contact points may only lie in the region where $g_{\Gamma}\left(d_{\Gamma}\right)$ is flat. This possibility, however, is ruled out by Theorem 3.4 (see the arguments on page 344 , and in particular the proof of (6.6) for further details). Thus, $x^{\star}$ cannot be an interior point. This proves that either $x^{\star} \in \partial \Omega$ or it lies on the boundary of the domain of $g_{\Gamma}\left(d_{\Gamma}\right)$. We now show that the latter possibility cannot hold. Indeed, on the boundary of the domain of $g_{\Gamma}\left(d_{\Gamma}\right)$ we have $g_{\Gamma}\left(d_{\Gamma}\right)=1$. On the other hand, if $x^{\star}$ lies on that boundary (but in the interior of $\Omega$ ), then

$$
u \leq 1=u\left(x^{\star}\right)=g_{\Gamma}\left(d_{\Gamma}\left(x^{\star}\right)\right)
$$

would give $\nabla u\left(x^{\star}\right)=0$. Let now $e$ be any direction pointing from $x^{\star}$ outside the domain of $g_{\Gamma}\left(d_{\Gamma}\right)$ and let $\hat{u}:=u-g_{\Gamma}\left(d_{\Gamma}\right)$. Then, by construction, $\hat{u}\left(x^{\star}-t e\right) \leq 0$ for any small positive $t$, while $\hat{u}\left(x^{\star}\right)=0$. Therefore, $\partial_{e} \hat{u}\left(x^{\star}\right) \geq 0$ for any outer derivative. If $e$ is taken to be the outer normal, however, then

$$
\partial_{e}\left(g_{\Gamma} \circ d_{\Gamma}\right)\left(x^{\star}\right)=g_{\Gamma}^{\prime}\left(d_{\Gamma}\left(x^{\star}\right)\right) \partial_{e} d_{\Gamma}\left(x^{\star}\right)=g_{\Gamma}^{\prime}\left(d_{\Gamma}\left(x^{\star}\right)\right)>0 .
$$

Collecting the above estimates, we have

$$
0>-\partial_{e}\left(g_{\Gamma} \circ d_{\Gamma}\right)\left(x^{\star}\right)=\partial_{e} \hat{u}\left(x^{\star}\right) \geq 0,
$$

and this contradiction show 10 that the contact point may only lie on $\partial \Omega$.

\footnotetext{
10 The reader will observe that the argument in 6.14 is indeed the same as on page 343
} 
The next result plays a crucial rôle, since it establishes that the zero mean curvature property of the limit surface is somehow already attained by solutions of $[1.5$, though in a weak viscosity sense.

Lemma 6.6. Let $l, \theta, \delta>0$ and $M_{1} \in \operatorname{Mat}((N-1) \times(N-1))$. Let $u$ be a weak Sobolev solution of 1.5$)$ in $[-l, l]^{N}$. Assume that $|u| \leq 1$ in $[-l, l]^{N}, u(0)=0$ and $u(x)<0$ for any $x=\left(x^{\prime}, x_{N}\right) \in[-l, l]^{N}$ so that

$$
x_{N}<\frac{\theta}{2 l^{2}} x^{\prime} \cdot M_{1} x^{\prime}+\frac{\theta}{l} \xi \cdot x^{\prime} .
$$

Assume also that (2.1) holds for $\omega=e_{N}$. Then there exist a universal constant $\delta_{0}>0$ and a function $\sigma:(0,1) \rightarrow(0,1)$ so that, if

$$
\delta \in\left(0, \delta_{0}\right], \quad \delta \leq \theta, \quad \theta / l \in(0, \sigma(\delta)], \quad\left\|M_{1}\right\| \leq 1 / \delta, \quad|\xi| \leq 1 / \delta,
$$

then $\operatorname{tr} M_{1} \leq \delta$.

Proof. We remark that, by our assumption, $l \geq \delta / \sigma(\delta)$ may and will be assumed to be a large quantity. Let $g_{l}$ and $\Psi^{y, l}$ be the functions defined in Lemma 5.1. Let also $\bar{c} \in(0,1 / 10)$ be such that

$$
T_{l / 4} \geq \bar{c} l
$$

Define also

$$
\Gamma_{1}:=\left\{x=\left(x^{\prime}, x_{N}\right) \in[-l, l]^{N}: x_{N}=\frac{\theta}{2 l^{2}} x^{\prime} \cdot M_{1} x^{\prime}+\frac{\theta}{l} \xi \cdot x^{\prime}\right\} .
$$

Let us make some elementary observations on the above paraboloid. First of all, by construction, $u$ is negative below $\Gamma_{1}$ in $[-l, l]^{N}$. Furthermore, by our assumptions,

$$
\Gamma_{1} \subseteq\left\{\left|x_{N}\right| \leq \mathrm{const} \theta / \delta\right\} \subseteq\left\{\left|x_{N}\right| \leq \mathrm{const} \sigma(\delta) l / \delta\right\} \subseteq\left\{\left|x_{N}\right| \leq \bar{c} l / 8\right\} .
$$

Therefore,

$$
x_{N}-\bar{c} l / 8 \leq d_{\Gamma_{1}}(x) \leq x_{N}+\bar{c} l / 8
$$

for any $x \in[-l, l]^{N}$.

Given $X \in \Gamma_{1}$ let $v_{X}$ be the normal direction to $\Gamma_{1}$ at $X$ pointing downwards. Let also

$$
\mathfrak{C}:=\left\{\left|x^{\prime}\right| \leq l / 4\right\} \times\left\{x_{N} \in[-l / 2, l / 8]\right\} .
$$

We claim that

$$
\mathfrak{C} \subseteq \bigcup_{X \in \Gamma_{1}} B_{(\bar{c}+1 / 4) l}\left(X+(l / 4) v_{X}\right)
$$

To prove this, take any $\mathfrak{x} \in \mathfrak{C}$ and let $X=X(\mathfrak{x}) \in \Gamma_{1}$ realize $d_{\Gamma_{1}}$. By 6.17), we have

$$
d_{\Gamma_{1}}(\mathfrak{x}) \in\left[-\frac{l}{2}-\frac{\bar{c} l}{8}, \frac{l}{8}+\frac{\bar{c} l}{8}\right] .
$$


This says in particular that $\left|d_{\Gamma_{1}}(\mathfrak{x})\right|<3 l / 4$, hence the defintion of $\mathfrak{C}$ implies that $X$ lies in the interior of $[-l, l]^{N}$ and therefore $\mathfrak{x}-X$ is orthogonal to $\Gamma_{1}$ at $X$, that is,

$$
\mathfrak{x}=X+\tau l v_{X}
$$

for a suitable $\tau \in \mathbb{R}$. Hence,

$$
d_{\Gamma_{1}}(\mathfrak{x})=-\tau l
$$

and

$$
\left|\mathfrak{x}-\left(X+(l / 4) v_{X}\right)\right|=|\tau-1 / 4| l .
$$

Then, by 6.20) and 6.19], we have

$$
\tau \in\left[-\frac{1}{8}-\frac{\bar{c}}{8}, \frac{1}{2}+\frac{\bar{c}}{8}\right]
$$

and so

$$
\tau-\frac{1}{4} \in\left[-\frac{3}{8}-\frac{\bar{c}}{8}, \frac{1}{4}+\frac{\bar{c}}{8}\right] \subset\left[-\frac{1}{4}-\frac{\bar{c}}{8}, \frac{1}{4}+\frac{\bar{c}}{8}\right] .
$$

This and 6.21$)$ imply that $\mathfrak{x} \in B_{(\bar{c}+1 / 4) l}\left(X+(l / 4) v_{X}\right)$. This proves 6.18).

We now observe that

$$
\frac{\theta\left\|M_{1}\right\|}{l^{2}} \leq \frac{\theta}{l^{2} \delta} \leq \frac{\sigma(\delta)}{l \delta} .
$$

The bound on the curvature of $\Gamma_{1}$ given in $(6.22)$ implies that, if $\sigma(\delta) / \delta$ is sufficiently small, then, given any $X \in \Gamma_{1}$, there exists a ball of radius $l / 4$ which touches $\Gamma_{1}$ from below at $X$.

The following is the decisive step towards the proof of the desired result. We claim that

$$
u(x) \leq g_{l / 4}\left(d_{\Gamma_{1}}(x)\right)
$$

for any $x \in \mathfrak{C}$. To prove $(6.23)$, first notice that, from (2.1) and Corollary 6.4, we infer that

$$
u(x)<\Psi^{(0, \ldots, 0,-l / 2), l / 4}(x), \quad \forall x \in B_{l / 4+T_{l / 4}}(0, \ldots, 0,-l / 2) .
$$

Then, for a given $X \in \Gamma_{1}$ we define

$$
X^{\prime}=X^{\prime}(X):=X+(l / 4) v_{X},
$$

where, as above, we denoted by $v_{X}$ the normal direction to $\Gamma_{1}$ at $X$ pointing downwards. In particular, from the above observation, $B_{l / 4}\left(X^{\prime}\right)$ touches $\Gamma_{1}$ from below at $X$. We now slide the surface $\Psi^{(0, \ldots, 0,-l / 2), l / 4}$ in the direction of the vector

$$
v=v(X):=X^{\prime}-(0, \ldots, 0,-l / 2),
$$

that is, we consider the surface $\Psi^{t}:=\Psi^{(0, \ldots, 0,-l / 2)+t v, l / 4}$ for $t>0$. We will show that

$$
\Psi^{t}(\mathfrak{x})>u(\mathfrak{x}) \text { for any } t \in[0,1) \text { and any } \mathfrak{x} \in B_{l / 4+T_{l / 4}}((0, \ldots, 0,-l / 2)+t v) .
$$

Indeed, let $t \in[0,1)$ be the first time at which $\Psi^{t}$ touches $u$. First of all, note that, since $t<1$, we have $u<0$ on $\partial B_{l / 4}((0, \ldots, 0,-l / 2)+t v)$, while $\Psi^{t}=0$ there. Therefore, $u$ 


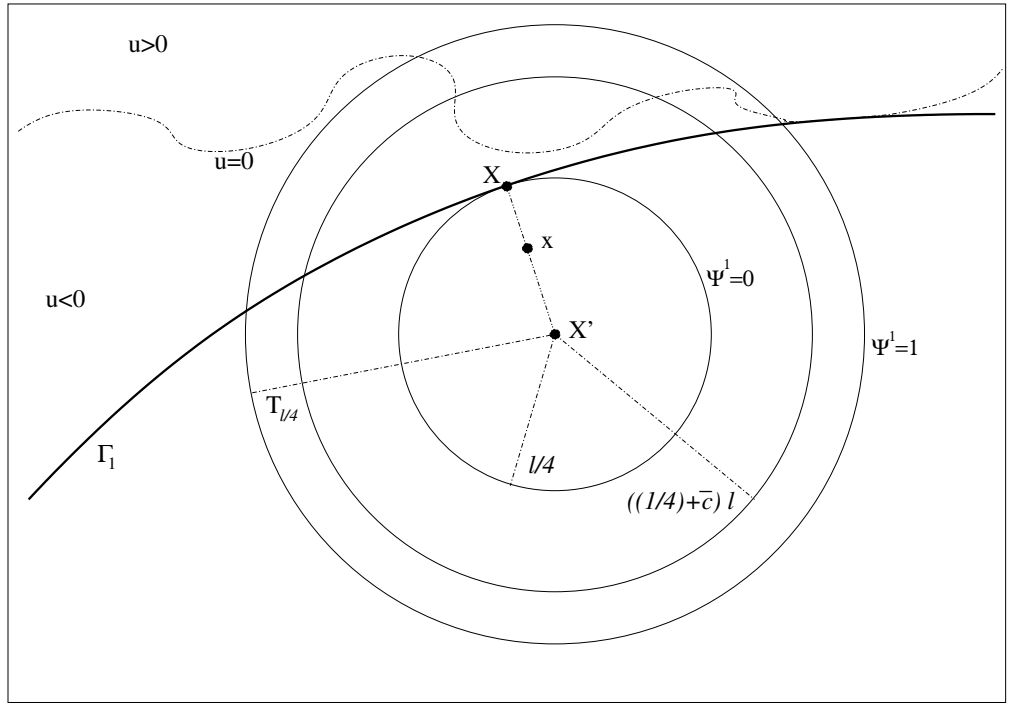

Touching between $\Psi^{1}$ and $\Gamma_{1}$

cannot be equal to $\Psi^{t}$, and no touching points occur on $\partial B_{l / 4}((0, \ldots, 0,-l / 2)+t v)$. On the other hand, Lemma 6.2 says that touching points cannot occur anywhere else. This proves (6.24).

We are now in a position to complete the proof of 6.23 by arguing as follows. We deduce from 6.24) that $\Psi^{1}(\mathfrak{x}) \geq u(\mathfrak{x})$ for any $\mathfrak{x} \in B_{l / 4+T_{l / 4}}\left(X^{\prime}\right)$, hence, a fortiori, for any $\mathfrak{x} \in B_{(1 / 4+\bar{c}) l}\left(X^{\prime}\right)$, thanks to $\sqrt{6.15}$. Therefore, taking now any $x \in \mathfrak{C}$ and letting $X^{\prime}$ be so that $x \in B_{(1 / 4+\bar{c}) l}\left(X^{\prime}\right)($ recall $(6.18)$ ), we have

$$
g_{l / 4}\left(d_{\Gamma_{1}}(x)\right)=g_{l / 4}\left(\left|x-X^{\prime}\right|-l / 4\right)=\Psi^{X^{\prime}, l / 4}(x)=\Psi^{1}(x) \geq u(x) .
$$

This proves 6.23.

We now complete the proof by supposing that $\operatorname{tr} M_{1}>\delta$ : under this assumption, by Lemma 5.2. $g_{\Gamma_{2}}\left(d_{\Gamma_{2}}\right)$ is a strict supersolution of $[1.5]$, where

$$
\begin{aligned}
\Gamma_{2} & :=\left\{x=\left(x^{\prime}, x_{N}\right) \in[-l, l]^{N}: x_{N}=\frac{\theta}{2 l^{2}} x^{\prime} \cdot M_{1} x^{\prime}+\frac{\theta}{l} \xi \cdot x^{\prime}-\frac{\varepsilon \delta}{2(N-1)}\left|x^{\prime}\right|^{2}\right\}, \\
\varepsilon & :=\frac{\theta}{2 l^{2}} .
\end{aligned}
$$

Note that

$$
\Gamma_{2} \subseteq\left\{\left|x_{N}\right| \leq \sigma(\delta)(\delta+1 / \delta) l\right\} \subseteq\left\{\left|x_{N}\right| \leq \bar{c} l / 8\right\} .
$$

Moreover, we claim that, if $\theta / l$ and $\delta$ are sufficiently small, then

$$
\begin{array}{ll}
h_{\Gamma_{2}}(s) \leq h_{l / 4}(s) & \text { if } s_{\delta, \varepsilon}<s \leq-1+\left(\delta \theta l^{-2}\right)^{1 / p}, \\
h_{\Gamma_{2}}(s) \geq h_{l / 4}(s) & \text { if } 1-\left(\delta \theta l^{-2}\right)^{1 / p} \leq s \leq 1 .
\end{array}
$$


Indeed, if $h_{\Gamma_{2}}(s)=0$, then 6.26 follows from item (i) in Lemma 5.1 If, on the contrary, $h_{\Gamma_{2}}(s)>0$ and $s \in\left[s_{\delta, \varepsilon},-1+(\delta \varepsilon)^{1 / p}\right]$, then, by the definitions of $h_{\Gamma_{2}}$ and $h_{l / 4}, 1.2$ and 5.23,

$$
\begin{aligned}
h_{\Gamma_{2}}(s)-h_{l / 4}(s) & \leq- \text { const } \delta \varepsilon+\frac{\text { const }}{l}(1+s)^{p}-\frac{\text { const }}{l} s_{l / 4}^{p}+h_{0}\left(-1+s_{l / 4}\right) \\
& \leq- \text { const } \frac{\delta \theta}{l^{2}}+\frac{\text { const }}{l}(1+s)^{p}+\text { const } s_{l / 4}^{p} \\
& \leq- \text { const } \frac{\delta \theta}{l^{2}}+\text { const } \frac{\delta \varepsilon}{l}+\text { const } s_{l / 4}^{p} \\
& =- \text { const } \frac{\delta \theta}{l^{2}}+\text { const } \frac{\delta \theta}{l^{3}}+\text { const } e^{- \text {const } l},
\end{aligned}
$$

which is negative for sufficiently large $l$, completing the proof of (6.26). To prove 6.27), use 1.2, 5.23 and the definitions of $h_{\Gamma_{2}}$ and $h_{l / 4}$ to deduce that, if $1-\left(\delta \theta l^{-2}\right)^{1 / p} \leq$ $s \leq 1$,

$$
\begin{aligned}
h_{\Gamma_{2}}(s)-h_{l / 4}(s) & \geq h_{0}(s)+\operatorname{const} \delta \varepsilon-h_{l / 4}(s) \\
& \geq \operatorname{const} \delta \varepsilon-h_{0}\left(1-s_{l / 4}\right)-\frac{\text { const }}{l}\left((1-s)^{p}+s_{l / 4}^{p}\right) \\
& \geq \operatorname{const} \delta \varepsilon-\operatorname{const} s_{l / 4}^{p}-\frac{\text { const }}{l}\left((1-s)^{p}+s_{l / 4}^{p}\right) \\
& \geq \operatorname{const} \delta \varepsilon-\operatorname{const} \frac{\delta \theta}{l^{3}}-\operatorname{const} e^{- \text {const } l} \\
& =\text { const } \frac{\delta \theta}{l^{2}}-\text { const } \frac{\delta \theta}{l^{3}}-\text { const } e^{- \text {const } l} .
\end{aligned}
$$

Taking $l$ large enough provides the proof of 6.27).

According to 6.26) and 6.27), the function $s \mapsto H_{\Gamma_{2}}(s)-H_{l / 4}(s)$ is increasing for $s \leq-1+\left(\delta \theta l^{-2}\right)^{1 / p}$ and decreasing for $s \geq 1-\left(\delta \theta l^{-2}\right)^{1 / p}$, therefore its maximum occurs in $\left[-1+\left(\delta \theta l^{-2}\right)^{1 / p}, 1-\left(\delta \theta l^{-2}\right)^{1 / p}\right]$, i.e.,

$$
\max _{s \in\left[s_{\delta, \varepsilon}, 1\right]}\left(H_{\Gamma_{2}}(s)-H_{l / 4}(s)\right)=\max _{s \in\left[-1+\left(\delta \theta l^{-2}\right)^{1 / p}, 1-\left(\delta \theta l^{-2}\right)^{1 / p}\right]}\left(H_{\Gamma_{2}}(s)-H_{l / 4}(s)\right) .
$$

Also, recalling the definition of $H_{0}$ in Lemma 5.1 if $s \in\left[0,1-\left(\delta \theta l^{-2}\right)^{1 / p}\right]$,

$$
H_{\Gamma_{2}}(s)=\int_{0}^{s} \frac{(p-1)^{1 / p} d \zeta}{\left(p h_{\Gamma_{2}}(\zeta)\right)^{1 / p}} \leq \int_{0}^{s} \frac{(p-1)^{1 / p} d \zeta}{\left(p h_{0}(\zeta)\right)^{1 / p}}=H_{0}(s),
$$

and analogously, if $s \in\left[-1+\left(\delta \theta l^{-2}\right)^{1 / p}, 0\right]$,

$$
-H_{\Gamma_{2}}(s)=\int_{s}^{0} \frac{(p-1)^{1 / p} d \zeta}{\left(p h_{\Gamma_{2}}(\zeta)\right)^{1 / p}} \geq \int_{s}^{0} \frac{(p-1)^{1 / p} d \zeta}{\left(p h_{0}(\zeta)\right)^{1 / p}}=-H_{0}(s) .
$$

Hence, from 6.29 and 6.30,

$$
H_{\Gamma_{2}}(s) \leq H_{0}(s)
$$


for any $s \in\left[-1+\left(\delta \theta l^{-2}\right)^{1 / p}, 1-\left(\delta \theta l^{-2}\right)^{1 / p}\right]$. Consequently, from $\left.\sqrt[5.4]{ }\right)$, if $s \in[-1+$ $\left.\left(\delta \theta l^{-2}\right)^{1 / p}, 1-\left(\delta \theta l^{-2}\right)^{1 / p}\right]$, then

$$
H_{\Gamma_{2}}(s) \leq H_{l / 4}(s)+\frac{\text { const }}{l} \log \frac{l^{2}}{\delta \theta} .
$$

Therefore, by 6.28),

$$
H_{\Gamma_{2}}(s) \leq H_{l / 4}(s)+\frac{\text { const }}{l} \log \frac{l^{2}}{\delta \theta}
$$

for any $s \in\left[s_{\delta, \varepsilon}, 1\right]$. Furthermore, by definition of $\Gamma_{1}$ and $\Gamma_{2}$, if $\left|x^{\prime}\right|=l / 4$, then

$$
d_{\Gamma_{2}}(x) \geq d_{\Gamma_{1}}(x)+c(\delta)
$$

for a suitable $c(\delta) \in(0,1)$. Hence, using 6.31) and taking $l$ appropriately large, with $s=g_{l / 4}\left(d_{\Gamma_{1}}(x)\right)$,

$$
\begin{aligned}
H_{\Gamma_{2}}\left(g_{l / 4}\left(d_{\Gamma_{1}}(x)\right)\right) & <H_{l / 4}\left(g_{l / 4}\left(d_{\Gamma_{1}}(x)\right)\right)+\frac{\text { const }}{l} \log \frac{l^{2}}{\delta \theta} \\
& =d_{\Gamma_{1}}(x)+\frac{\text { const }}{l} \log \frac{l^{2}}{\delta \theta} \leq d_{\Gamma_{2}}(x)
\end{aligned}
$$

provided $g_{l / 4}\left(d_{\Gamma_{1}}(x)\right) \geq s_{\delta, \varepsilon}$ and $\left|x^{\prime}\right|=l / 4$; therefore, since $H_{\Gamma_{2}}$ is strictly increasing in $\left[s_{\delta, \varepsilon}, 1\right]$,

$$
g_{l / 4}\left(d_{\Gamma_{1}}(x)\right)<g_{\Gamma_{2}}\left(d_{\Gamma_{2}}(x)\right)
$$

for any $x$ so that $g_{l / 4}\left(d_{\Gamma_{1}}(x)\right) \geq s_{\delta, \varepsilon}$ and $\left|x^{\prime}\right|=l / 4$. Of course, if $g_{l / 4}\left(d_{\Gamma_{1}}(x)\right)<s_{\delta, \varepsilon}$, then 6.32 holds since $g_{\Gamma_{2}} \geq s_{\delta, \varepsilon}$ by construction (recall item (ii) of Lemma 5.2. Thus,

$$
g_{l / 4}\left(d_{\Gamma_{1}}(x)\right)<g_{\Gamma_{2}}\left(d_{\Gamma_{2}}(x)\right), \quad \forall\left|x^{\prime}\right|=l / 4,
$$

provided that $d_{\Gamma_{1}}(x)$ is in the domain of $g_{l / 4}$ and $d_{\Gamma_{2}}(x)$ in the domain of $g_{\Gamma_{2}}$. Notice, however, that the first of these conditions is implied by the second:

$$
\text { if } d_{\Gamma_{2}}(x) \text { is in the domain of } g_{\Gamma_{2}} \text {, then } d_{\Gamma_{1}}(x) \text { is in the domain of } g_{l / 4} \text {. }
$$

To prove this, take $x$ so that $d_{\Gamma_{2}}(x)$ is in the domain of $g_{\Gamma_{2}}$. Then, by Lemma 5.2 and our choice of parameters,

$$
d_{\Gamma_{2}}(x) \leq C_{0}(\delta) \log \frac{l^{2}}{\theta},
$$

and thus, from 6.15, 6.16 and 6.25), we deduce that

$$
d_{\Gamma_{1}}(x) \leq d_{\Gamma_{2}}(x)+\frac{\bar{c} l}{4} \leq C_{0}(\delta) \log \frac{l^{2}}{\theta}+\frac{\bar{c} l}{4} \leq \frac{\bar{c} l}{2} \leq T_{l / 4},
$$

which says that $d_{\Gamma_{1}}(x)$ is in the domain of $g_{l / 4}$, thus proving 6.34). 
Now, 6.33, 6.23 and 6.34 imply that

$$
u(x)<g_{\Gamma_{2}}\left(d_{\Gamma_{2}}(x)\right)
$$

for any $x$ so that $\left|x^{\prime}\right|=l / 4$ and $d_{\Gamma_{2}}(x)$ is in the domain of $g_{\Gamma_{2}}$.

With these estimates, we are now ready to deduce the contradiction that will finish the proof of the desired result. To this end, we slide $g_{\Gamma_{2}}\left(d_{\Gamma_{2}}\right)$ (as constructed in Lemma 5.2) in the $e_{N}$-direction till we touch $u$ in $\mathfrak{C}$, i.e., we consider, for $t \in \mathbb{R}$,

$$
g^{t}(x):=g_{\Gamma_{2}}\left(d_{\Gamma_{2}}\left(x-t e_{N}\right)\right) .
$$

If we denote by $\mathcal{D}_{0}$ the domain of $g_{\Gamma_{2}}\left(d_{\Gamma_{2}}\right)$, then Lemma 5.2 shows that $\mathcal{D}_{0}$ is the subgraph of a paraboloid, namely

$$
\mathcal{D}_{0}=\left\{d_{\Gamma_{2}}(x) \leq T_{\varepsilon, \delta}\right\}
$$

Also, on the top of the paraboloid, $g_{\Gamma_{2}}\left(d_{\Gamma_{2}}\right)$ takes value 1, i.e., if $d_{\Gamma_{2}}(x)=T_{\varepsilon, \delta}$ then $g_{\Gamma_{2}}\left(d_{\Gamma_{2}}(x)\right)=1$. Notice that, with this notation, $g^{t}$ is defined in $\mathcal{D}_{t}:=\mathcal{D}_{0}+t e_{N}$ and $g^{t}$ takes value 1 on the top of $\mathcal{D}_{t}$. Thus, if $t \ll 0$, then $g^{t}>u$ in $\mathcal{D}_{t} \cap \mathfrak{C}$, since $u<0$ below $\Gamma_{1}$. On the other hand,

$$
g^{0}(0)=g_{\Gamma_{2}}\left(d_{\Gamma_{2}}(0)\right)=g_{\Gamma_{2}}(0)=0=u(0),
$$

therefore, there is a time $t \leq 0$ of first touch of $g^{t}$ and $u$ in $\mathcal{D}_{t} \cap \mathfrak{C}$. Hence, in light of Lemma 6.5. contact points may only happen either on the lateral side of the cylinder $\mathfrak{C}$ (i.e., on $\left|x^{\prime}\right|=l / 4$ ) or on its bottom (i.e., on $x_{N}=-l / 2$; the case $x_{N}=l / 8$, corresponding to the upper face of the cylinder $\mathfrak{C}$ cannot hold because $t \leq 0$, hence $\mathcal{D}_{t}$ lies way below $x_{N}=l / 8$, due to 6.36 ).

We now exclude the possibility of touching at $x_{N}=-l / 2$ by arguing as follows. By applying 6.23, 6.17) and the fact that $g_{l / 4}$ is constant in $(-\infty,-l / 8]$, we deduce that, if $x_{N}=-l / 2$, then

$$
\begin{aligned}
u(x) & \leq g_{l / 4}\left(d_{\Gamma_{1}}(x)\right) \leq g_{l / 4}\left(x_{N}+\frac{\bar{c} l}{8}\right)=g_{l / 4}\left(-\frac{l}{2}+\frac{\bar{c} l}{8}\right) \leq g_{l / 4}\left(-\frac{l}{8}\right) \\
& =-1+e^{\text {const } l}<s_{\delta, \varepsilon} \leq g^{t}(x),
\end{aligned}
$$

which rules out the possibility of touching at $x_{N}=-l / 2$.

Therefore, a contact point $x^{\star} \in \mathcal{D}_{t} \cap \mathfrak{C}$ between $u$ and $g^{t}$ does occur when $\left|x^{\prime}\right|=l / 4$. Notice now that, from Lemma 4.8 .

$$
d_{\Gamma_{2}}\left(x^{\star}-t e_{N}\right) \geq d_{\Gamma_{2}}\left(x^{\star}\right) .
$$

But then, since $g_{\Gamma_{2}}$ is non-decreasing, we deduce from 6.35 that

$$
g_{\Gamma_{2}}\left(d_{\Gamma_{2}}\left(x^{\star}-t e_{N}\right)\right)=g^{t}\left(x^{\star}\right)=u\left(x^{\star}\right)<g_{\Gamma_{2}}\left(d_{\Gamma_{2}}\left(x^{\star}\right)\right) \leq g_{\Gamma_{2}}\left(d_{\Gamma_{2}}\left(x^{\star}-t e_{N}\right)\right) .
$$

This contradiction concludes the proof of Lemma 6.6 


\section{Proof of Theorem 2.2}

The proof of Theorem 2.2 can now be completed as follows. We will apply Lemma 6.6 with the following choice of parameters:

$$
l:=\frac{\mathfrak{d}}{\sqrt{\varepsilon \operatorname{tr} M}}, \quad \delta:=\theta:=\mathfrak{d}^{2}, \quad M_{1}:=\frac{1}{\operatorname{tr} M} M, \quad \xi:=0 .
$$

If the claim of Theorem 2.2 were false, by scaling back and using the above parameters, we would infer that $\Gamma_{1}$ touches from below the zero level set of $u$ inside $[-l, l]^{N}$, where

$$
\Gamma_{1}=\left\{x=\left(x^{\prime}, x_{N}\right) \in \mathbb{R}^{N-1} \times \mathbb{R}: x_{N}=\frac{\theta}{2 l^{2}} x^{\prime} \cdot M_{1} x^{\prime}+\frac{\theta}{l} \xi \cdot x^{\prime}\right\} .
$$

By Lemma 6.6, we gather that $1>\delta \geq \operatorname{tr} M_{1}=1$, which is the contradiction that proves Theorem 2.2.

\section{Proof of Theorem 2.1}

We are now in a position to prove Theorem 2.1. Let $x^{\star}$ be a point where $\mathcal{S}$ admits a tangent plane. With no loss of generality, we may assume that the normal vector to $\mathcal{S}$ at $x^{\star}$ is $e_{N}$. Let $P$ be a paraboloid touching from below. We will show that the mean curvature of $P$ is non-positive; an analogous proof gives that, if $P$ is a paraboloid touching from above, then its mean curvature is non-negative, and this ends the proof of Theorem 2.1 .

By construction, if $P$ touches $\mathcal{S}$ from below at $x^{\star}$, then

$$
P=\left\{\left(x^{\prime}, x\right) \in \mathbb{R}^{N-1} \times \mathbb{R}: x_{N}=\frac{1}{2}\left(x^{\prime}-\left(x^{\star}\right)^{\prime}\right) \cdot \mathcal{M}\left(x^{\prime}-\left(x^{\star}\right)^{\prime}\right)+x_{N}^{\star}\right\}
$$

for some $\mathcal{M} \in \operatorname{Mat}((N-1) \times(N-1))$. Let $\mathbb{I}_{N-1}$ be the $(N-1) \times(N-1)$ identity matrix and set

$$
\widehat{\mathcal{M}}:=\mathcal{M}-\frac{\operatorname{tr} \mathcal{M}}{2(N-1)} \mathbb{I}_{N-1} \text {. }
$$

Notice that

$$
\operatorname{tr} \widehat{\mathcal{M}}=\frac{1}{2} \operatorname{tr} \mathcal{M}
$$

Let us also define

$$
\mathfrak{P}:=\left\{\left(x^{\prime}, x\right) \in \mathbb{R}^{N-1} \times \mathbb{R}: x_{N}=\frac{1}{2}\left(x^{\prime}-\left(x^{\star}\right)^{\prime}\right) \cdot \widehat{\mathcal{M}}\left(x^{\prime}-\left(x^{\star}\right)^{\prime}\right)+x_{N}^{\star}\right\} .
$$

Assume, by contradiction, that $\operatorname{tr} \mathcal{M}>0$. Then $\operatorname{tr} \widehat{\mathcal{M}}>0$ in light of 8.2 , and $\mathfrak{P}$ also touches $\mathcal{S}$ from below at $x^{\star}$. Let $r \in(0,1)$, to be chosen suitably small later, and consider the cube $Q:=\left\{\left|x-x^{\star}\right|_{\infty} \leq r\right\}$. Notice that, by the tangency of $\left\{x_{N}=x_{N}^{\star}\right\}$ and $\mathcal{S}$, if $r$ is small enough, we have

$$
\mathcal{S} \cap Q \subseteq\left\{\left|x_{N}-x_{N}^{\star}\right| \leq r / 4\right\}
$$


Also, by 2.2, if we fix any $\eta>0$, we have

$$
\operatorname{dist}(x, \mathcal{S}) \leq \eta
$$

for any $x \in\left\{u_{\varepsilon}=0\right\} \cap B_{1}\left(x^{\star}\right)$, provided $\varepsilon$ is small enough. We also claim that

$$
B_{\eta}\left(x^{\star}\right) \cap\left\{u_{\varepsilon}=0\right\} \neq \emptyset
$$

if $\varepsilon$ is small enough. To prove this, assume that, say, $u_{\varepsilon}<0$ in $B_{\eta}\left(x^{\star}\right)$. Then, since $u_{\varepsilon}$ converges in $L_{\text {loc }}^{1}$ to $\chi:=\chi_{\mathcal{E}}-\chi_{\mathbb{R}^{N} \backslash \mathcal{E}}$, we have

$$
\begin{aligned}
0 & \geq \lim _{\varepsilon \rightarrow 0^{+}} \int_{B_{\eta}\left(x^{\star}\right)}\left|u_{\varepsilon}-\chi\right|=\lim _{\varepsilon \rightarrow 0^{+}} \int_{B_{\eta}\left(x^{\star}\right) \cap \mathcal{E}}\left|u_{\varepsilon}-1\right|+\int_{B_{\eta}\left(x^{\star}\right) \cap\left(\mathbb{R}^{N} \backslash \mathcal{E}\right)}\left|u_{\varepsilon}+1\right| \\
& \geq \mathfrak{L}\left(B_{\eta}\left(x^{\star}\right) \cap \mathcal{E}\right),
\end{aligned}
$$

where $\mathfrak{L}$ is the $N$-dimensional Lebesgue measure, so

$$
\mathfrak{L}\left(B_{\eta}\left(x^{\star}\right) \cap \mathcal{E}\right)=0 ;
$$

this contradicts 2.3 and proves 8.5 .

We will now consider the touching of a suitable sliding of $\mathfrak{P}$ with $\left\{u_{\varepsilon}=0\right\}$ in $Q$. In order to formalize the argument, define

$$
\mathfrak{P}_{t}:=\left\{\left(x^{\prime}, x\right) \in \mathbb{R}^{N-1} \times \mathbb{R}: x_{N}=\frac{1}{2}\left(x^{\prime}-\left(x^{\star}\right)^{\prime}\right) \cdot \widehat{\mathcal{M}}\left(x^{\prime}-\left(x^{\star}\right)^{\prime}\right)+x_{N}^{\star}-t\right\},
$$

and let $t \in \mathbb{R}$ be so that $\mathfrak{P}_{t} \cap\left\{u_{\varepsilon}=0\right\} \cap Q \neq \emptyset$, while $\mathfrak{P}_{s} \cap\left\{u_{\varepsilon}=0\right\} \cap Q=\emptyset$ for any $s>t$. Notice that, from 8.4 and $(8.5)$,

$$
|t| \leq 2 \eta
$$

Let $x^{\sharp} \in \mathfrak{P}_{t} \cap\left\{u_{\varepsilon}=0\right\} \cap Q$. We now show that $x^{\sharp}$ is in the interior of $Q$; more precisely,

$$
x^{\sharp} \in\left\{\left|x-x^{\star}\right|_{\infty} \leq r / 2\right\} .
$$

First of all, by 8.3 and 8.4 ,

$$
\left\{u_{\varepsilon}=0\right\} \cap Q \subseteq\left\{\left|x_{N}-x_{N}^{\star}\right| \leq r / 2\right\}
$$

provided $\varepsilon$ is small enough. Hence, to prove 8.7 , it remains to show that $\left|x_{i}^{\sharp}-x_{i}^{\star}\right| \leq r / 2$ for $1 \leq i \leq N-1$.

For this, recalling 8.4, let $\tilde{x} \in \mathcal{S}$ be so that

$$
\left|\tilde{x}-x^{\sharp}\right| \leq \eta .
$$

Then, since $\mathcal{S}$ is above $P$,

$$
\tilde{x}_{N} \geq \frac{1}{2}\left(\tilde{x}^{\prime}-\left(x^{\star}\right)^{\prime}\right) \cdot \mathcal{M}\left(\tilde{x}^{\prime}-\left(x^{\star}\right)^{\prime}\right)+x_{N}^{\star} .
$$


Since, on the other hand, $x^{\sharp} \in \mathfrak{P}_{t}$,

$$
x_{N}^{\sharp}=\frac{1}{2}\left(\left(x^{\sharp}\right)^{\prime}-\left(x^{\star}\right)^{\prime}\right) \cdot \widehat{\mathcal{M}}\left(\left(x^{\sharp}\right)^{\prime}-\left(x^{\star}\right)^{\prime}\right)+x_{N}^{\star}-t,
$$

which, from 8.1 and $(8.6$, implies

$$
x_{N}^{\sharp} \leq-\frac{\operatorname{tr} \mathcal{M}}{4(N-1)}\left|\left(x^{\sharp}\right)^{\prime}-\left(x^{\star}\right)^{\prime}\right|^{2}+\frac{1}{2}\left(\left(x^{\sharp}\right)^{\prime}-\left(x^{\star}\right)^{\prime}\right) \cdot \mathcal{M}\left(\left(x^{\sharp}\right)^{\prime}-\left(x^{\star}\right)^{\prime}\right)+x_{N}^{\star}+2 \eta \text {. }
$$

Subtracting (8.10) and (8.11), and making use of (8.9), one gets

$$
\begin{aligned}
\text { const } \eta \geq & \tilde{x}_{N}-x_{N}^{\sharp} \\
\geq & \frac{\operatorname{tr} \mathcal{M}}{4(N-1)}\left|\left(x^{\sharp}\right)^{\prime}-\left(x^{\star}\right)^{\prime}\right|^{2}+\frac{1}{2}\left(\tilde{x}^{\prime}-\left(x^{\star}\right)^{\prime}\right) \cdot \mathcal{M}\left(\tilde{x}^{\prime}-\left(x^{\star}\right)^{\prime}\right) \\
& -\frac{1}{2}\left(\left(x^{\sharp}\right)^{\prime}-\left(x^{\star}\right)^{\prime}\right) \cdot \mathcal{M}\left(\left(x^{\sharp}\right)^{\prime}-\left(x^{\star}\right)^{\prime}\right)-2 \eta \\
\geq & \frac{\operatorname{tr} \mathcal{M}}{4(N-1)}\left|\left(x^{\sharp}\right)^{\prime}-\left(x^{\star}\right)^{\prime}\right|^{2}-\operatorname{const}(1+\|\mathcal{M}\|) \eta .
\end{aligned}
$$

Since $\operatorname{tr} \mathcal{M}>0$, this shows in particular that $\left|x_{i}^{\sharp}-x_{i}^{\star}\right| \leq r / 2$ for $1 \leq i \leq N-1$, provided $\eta$ (and so $\varepsilon$ ) is suitably small, thus completing the proof of $(8.7)$.

With no loss of generality, we may now assume that $x^{\sharp}=0$. Notice that, with this assumption, by (8.7), $\mathfrak{P}_{t}$ touches $\left\{u_{\varepsilon}=0\right\}$ from below in $\left\{|x|_{\infty} \leq r / 2\right\}$. Hence, either $u_{\varepsilon}<0$ or $u_{\varepsilon}>0$ below $\mathfrak{P}_{t}$ in $[-r / 2, r / 2]^{N}$; we will consider the first possibility, the second being analogous. Namely, we assume that

$$
u_{\varepsilon}<0 \quad \text { below } \mathfrak{P}_{t} \text { in }[-r / 2, r / 2]^{N} .
$$

Also, since $0 \in \mathfrak{P}_{t}$, the equation of $\mathfrak{P}_{t}$ takes the form

$$
x_{N}=\frac{1}{2} x^{\prime} \cdot \widehat{\mathcal{M}} x^{\prime}+V \cdot x^{\prime}
$$

with

$$
|V| \leq \text { const }\|\widehat{\mathcal{M}}\| r \text {. }
$$

We now apply Lemma 6.6 with the following choices: let $\delta \in(0,1)$ be a fixed small quantity, $M_{1}:=\widehat{\mathcal{M}} / \operatorname{tr} \widehat{\mathcal{M}}, l:=r /(2 \varepsilon), \theta:=\varepsilon l^{2} \operatorname{tr} \widehat{\mathcal{M}}, \xi:=l V / \theta$. Let us check that the hypotheses of Lemma 6.6 are fulfilled in the setting above if we choose $r$ small (in dependence on a fixed, small $\delta$ ) and $\varepsilon$ small (in dependence on $\delta$ and $r$ ). Indeed,

$$
\theta=\mathrm{const} \frac{r^{2} \operatorname{tr} \widehat{\mathcal{M}}}{\varepsilon}
$$

is greater than $\delta$ if $\varepsilon$ is small enough. On the other hand,

$$
\frac{\theta}{l}=\varepsilon l \operatorname{tr} \widehat{\mathcal{M}}=\text { const } r \operatorname{tr} \widehat{\mathcal{M}}
$$


is less than $\sigma(\delta)$ if $r$ is small. Furthermore, $\left\|M_{1}\right\|$ is obviously less than $1 / \delta$ if $\delta$ is small enough and also

$$
|\xi| \leq \mathrm{const} \frac{\|\widehat{\mathcal{M}}\| r l}{\theta}=\mathrm{const} \frac{\left\|M_{1}\right\| r}{\varepsilon l}=\mathrm{const}\left\|M_{1}\right\|,
$$

which is less than $1 / \delta$ if $\delta$ is small. The last hypothesis to check in order to use Lemma 6.6 is that

$$
u<0
$$

for any $x \in[-l, l]^{N}$ with

$$
x_{N}<\frac{\theta}{2 l^{2}} x^{\prime} \cdot M_{1} x^{\prime}+\frac{\theta}{l} \xi \cdot x^{\prime} .
$$

To prove this, let $x$ be as above and set $y:=\varepsilon x$. Then

$$
|y|_{\infty} \leq \varepsilon l \leq r / 2
$$

and

$$
y_{N}<\frac{\theta}{2 l^{2} \varepsilon} y^{\prime} \cdot M_{1} y^{\prime}+\frac{\theta}{l} \xi \cdot y^{\prime}=\frac{1}{2} y^{\prime} \cdot \widehat{\mathcal{M}} y^{\prime}+V \cdot y^{\prime},
$$

hence $y$ is below $\mathfrak{P}_{t}$ thanks to 8.13 , and therefore, recalling 8.12 , we deduce that

$$
0>u_{\varepsilon}(y)=u(x) \text {. }
$$

This proves 8.14 . Since the assumptions of Lemma 6.6 are fulfilled, we deduce from Lemma 6.6 that

$$
1>\delta \geq \operatorname{tr} M_{1}=1 .
$$

This contradiction, caused by the assumption that $\operatorname{tr} \mathcal{M}>0$, shows that the mean curvature of $P$ must be non-positive. This completes the proof of Theorem 2.1

\section{Comments on the decay property (2.1) and proof of Theorem 2.3}

In this section we assume that $h_{0}$ admits a $C^{1}$ extension to a neighborhood of $[-1,1]$.

We would like to show that conditions of type (2.1) are somehow natural. First of all, a condition of this kind is necessary to avoid, for instance, periodic one-dimensional solutions. Also, Class A minimizers of $\mathcal{F}$ fulfill (2.1). This follows from some density estimates for minimizers of $\mathcal{F}$, proved in [16]. In our setting ${ }^{11}$, we may state this result as follows. We say that $u$ is a local minimize $\sqrt{12}$ for $\mathcal{F}$ in some bounded domain $\Omega$ if $\mathcal{F}_{\Omega}(u) \leq$ $\mathcal{F}_{\Omega}(u+\varphi)$ for any smooth function $\varphi$ supported in $\Omega$. Then the following result holds:

\footnotetext{
11 In (part of) [16] a uniform Lipschitz assumption on $h_{0}$ is assumed. Such a condition is fulfilled here, because, for any $\xi \in\left(-1,-1+\theta^{*}\right)$ and $\zeta \in\left(1-\theta^{*}, 1\right)$, we have

$$
\left|h_{0}^{\prime}(\xi)\right|=h_{0}^{\prime}(\xi) \leq h_{0}^{\prime}\left(-1+\theta^{*}\right) \quad \text { and } \quad\left|h_{0}^{\prime}(\zeta)\right|=-h_{0}^{\prime}(\zeta) \leq-h_{0}^{\prime}\left(1-\theta^{*}\right) .
$$

Hence, $\sup _{(-1,1)}\left|h_{0}^{\prime}\right| \leq$ const.

12 The reader will note that, with this definition, a Class A minimizer is simply a local minimizer in any domain.
} 
Theorem 9.1 ([16]). Let $u$ be a local minimizer of $\mathcal{F}$ in a domain $\Omega$ such that $|u| \leq 1$. Fix $\epsilon \in(0,1)$ and suppose $x \in\{-1+\epsilon<u<1-\epsilon\}$. Then there exist positive $r_{0}(\epsilon)$ and $c(\epsilon)$ such that

$$
\mathfrak{L}\left(B_{r}(x) \cap\{u>-1+\epsilon\}\right) \geq c(\epsilon) r^{N} \quad \text { and } \quad \mathfrak{L}\left(B_{r}(x) \cap\{u<1-\epsilon\}\right) \geq c(\epsilon) r^{N}
$$

for any $r \geq r_{0}(\epsilon)$, where $\mathfrak{L}$ is the $N$-dimensional Lebesgue measure, provided $B_{r+1}(x)$ $\subset \Omega$.

Further details on the above result are given in Theorem 1.1 of [16]. This implies that local (and hence Class A) minimizers satisfy (2.1):

Corollary 9.2. Any local minimizer of $\mathcal{F}$ in $[-l, l]^{N}$ satisfies 2.1 for any $\omega \in \mathrm{S}^{N-1}$, provided that $l$ is large enough.

Proof. Suppose that

$$
\{u=0\} \cap(\{|\omega \cdot x| \leq l\} \times\{|x-(\omega \cdot x) \omega| \leq l\}) \subset\left\{\omega \cdot x \geq-c_{1}^{\star} l\right\}
$$

and let $x=\left(x^{\prime}, x_{N}\right) \in \mathbb{R}^{N-1} \times \mathbb{R}$ satisfy $\omega \cdot x<-c_{2}^{\star} l$ and $|x-(\omega \cdot x) \omega| \leq c_{3}^{\star} l$. Fix $\epsilon>0$, and assume by contradiction $u(x)>-1+\epsilon$. By Theorem 9.1 with $l$ sufficiently large with respect to $r_{0}(\epsilon)$, we see that $B_{\left(c_{2}^{\star}-c_{1}^{\star}\right) l / 2}(x)$ contains points where $u=0$. This contradiction shows that 2.1.

The proof of Theorem 2.3 is now completed via Corollary 9.2 .

Thanks to Lemma 6.6 and Corollary 9.2 we can now state the following result, which will be of use in the forthcoming paper [22]:

Lemma 9.3. Let $l, \theta, \delta>0$ and $M_{1} \in \operatorname{Mat}((N-1) \times(N-1))$. Let $u$ be a local minimizer of $\mathcal{F}$ in $[-l, l]^{N}$, with $|u| \leq 1$ in $[-l, l]^{N}, u(0)=0$ and $u(x)<0$ for any $x=\left(x^{\prime}, x_{N}\right) \in[-l, l]^{N}$ so that

$$
x_{N}<\frac{\theta}{2 l^{2}} x^{\prime} \cdot M_{1} x^{\prime}+\frac{\theta}{l} \xi \cdot x^{\prime}
$$

Then there exist a universal constant $\delta_{0}>0$ and a function $\sigma:(0,1) \rightarrow(0,1)$ so that, if

$$
\delta \in\left(0, \delta_{0}\right], \quad \delta \leq \theta, \quad \theta / l \in(0, \sigma(\delta)], \quad\left\|M_{1}\right\| \leq 1 / \delta, \quad|\xi| \leq 1 / \delta
$$

then $\operatorname{tr} M_{1} \leq \delta$

To end this paper, we note that, as a matter of fact, the barriers constructed here also show that Class A minimizers approach \pm 1 exponentially fast, once the zero level set is under control. Though this result is not explicitly used here, we believe it is useful to clarify the picture of Class A minimizers: 
Lemma 9.4. Let $u \in W_{\text {loc }}^{1, p}\left(\mathbb{R}^{N}\right)$ be a weak Sobolev solution of 1.5 so that $u(x)<$ $-1+\theta^{*}$ for any $x \in \mathbb{R}^{N}$ with $x_{N} \leq-\kappa$ for some $\kappa>0$. Then

$$
\begin{array}{ll}
u(x) \leq-1+\theta^{*} e^{\kappa^{\prime}\left(x_{N}+\kappa\right)} & \text { if } x_{N} \leq-\kappa, \\
u(x) \geq 1-\theta^{*} e^{\kappa^{\prime}\left(\kappa-x_{N}\right)} & \text { if } x_{N} \geq \kappa,
\end{array}
$$

for a suitable $\kappa^{\prime}>0$.

What is more, let $u$ be a Class A minimizer for $\mathcal{F}$ with $|u| \leq 1$. Let $\theta>0$ and assume that $u(x)<0$ if $x_{N} \leq-\theta$, and $u(x)>0$ if $x_{N} \geq \theta$. Then there exist $\kappa_{1}, \kappa_{2}>0$, depending only on $\theta$ and on universal constants, so that

$$
\begin{array}{ll}
u(x) \leq-1+\theta^{*} e^{\kappa_{1}\left(x_{N}+\kappa_{2}\right)} & \text { if } x_{N} \leq-\kappa_{2}, \\
u(x) \geq 1-\theta^{*} e^{\kappa_{1}\left(\kappa_{2}-x_{N}\right)} & \text { if } x_{N} \geq \kappa_{2} .
\end{array}
$$

Proof. Let $u$ be a Class A minimizer. By arguing as in Corollary 9.2, one easily deduces from Theorem 9.1 and the inclusion $\{u=0\} \subseteq\left\{\left|x_{N}\right| \leq \theta\right\}$, that

$$
u(x)<-1+\theta^{*} \quad \text { for any } x \in \mathbb{R}^{N} \text { with } x_{N} \leq-\kappa_{2},
$$

for a suitable $\kappa_{2}$ (depending only on $\theta, \theta^{*}$ and universal constants). This shows that the claim in 9.3 reduces to the one in 9.2. Now, for $(x, t) \in \mathbb{R}^{N} \times\left[0, \theta^{*}\right]$ let

$$
\beta^{(t)}(x):=-1+\theta^{*} e^{\kappa_{1}\left(\kappa_{2}+x_{N}\right)}+t .
$$

Notice that, by 9.4 and 9.5 ,

$$
\beta^{(t)} \geq-1+\theta^{*}>u
$$

in $\left\{x_{N} \leq-\kappa_{2}\right\}$. Also, with a straightforward computation, recalling (1.2), one gets

$$
\begin{aligned}
\Delta_{p} \beta^{(t)} & =\Delta_{p} \beta^{(0)}=(p-1) \kappa_{1}^{\mathrm{const}}\left(1+\beta^{(0)}\right)^{p-1} \\
& \leq(p-1) \kappa_{1}^{\mathrm{const}}\left(1+\beta^{(t)}\right)^{p-1} \leq h_{0}^{\prime}\left(\beta^{(t)}\right)
\end{aligned}
$$

provided $\kappa_{1}$ is small enough. Then we slide $\beta^{(t)}$, decreasing $t$ from $\theta^{*}$ towards 0 until we possibly touch $u$ in $\left\{x_{N} \leq-\kappa_{2}\right\}$. We prove in fact that no touching occurs until $t=0$, that is,

$$
\beta^{(t)}>u
$$

for any $x \in \mathbb{R}^{N}$ with $x_{N} \leq-\kappa_{2}$ and any $t \in\left(0, \theta^{*}\right]$. To prove 9.7$)$, assume the converse; then, in light of (9.4), a touching point occurs at some $x^{\star}$ with $x_{N}^{\star}<-\kappa_{2}$.

Note now that

$$
\left|\nabla \beta^{(t)}(x)\right|=\theta^{*} \kappa_{1} e^{\kappa_{1}\left(\kappa_{2}+x_{N}\right)}>0 .
$$

In particular, if we consider the set $B_{\rho}\left(x^{\star}\right)$ with $\rho:=-x_{N}^{\star}-\kappa_{2}$ it follows that $u<\beta^{(t)}$ at least at some point on $\partial B_{\rho}\left(x^{\star}\right)$. Therefore, by Theorem 3.3 .

$$
\beta^{(t)}>u \quad \text { in } B_{\rho}\left(x^{\star}\right),
$$

which is a contradiction. This proves 9.7 . From this, $u \leq \beta^{(0)}$, which proves the first estimate in 9.2 - -9.3 . The second one can be proven in a similar way. 


\section{References}

[1] Alberti, G., Ambrosio, L., Cabré, X.: On a long-standing conjecture of E. De Giorgi: symmetry in 3D for general nonlinearities and a local minimality property. Acta Appl. Math. 65, 9-33 (2001) Zbl pre01657982 MR 1843784

[2] Ambrosio, L., Cabré, X.: Entire solutions of semilinear elliptic equations in $\mathbb{R}^{3}$ and a conjecture of De Giorgi. J. Amer. Math. Soc. 13, 725-739 (2000) Zbl 0968.35041 MR 1775735

[3] Antman, S. S.: Nonuniqueness of equilibrium states for bars in tension. J. Math. Anal. Appl. 44, 333-349 (1973) Zbl 0267.73031 MR 0334637

[4] Bouchitté, G.: Singular perturbations of variational problems arising from a two-phase transition model. Appl. Math. Optim. 21, 289-314 (1990) Zbl 0695.49003 MR 1036589

[5] Caffarelli, L. A., Córdoba, A.: Uniform convergence of a singular perturbation problem. Comm. Pure Appl. Math. 48, 1-12 (1995) Zbl 0829.49013 MR 1310848

[6] Damascelli, L.: Comparison theorems for some quasilinear degenerate elliptic operators and applications to symmetry and monotonicity results. Ann. Inst. H. Poincaré Anal. Non Linéaire 15, 493-516 (1998) Zbl 0911.35009 MR 1632933

[7] De Giorgi, E.: Convergence problems for functionals and operators. In: Proc. International Meeting on Recent Methods in Nonlinear Analysis (Rome, 1978), Pitagora, Bologna, 131-188 (1979) Zbl 0405.49001 MR 0533166

[8] DiBenedetto, E.: $C^{1+\alpha}$ local regularity of weak solutions of degenerate elliptic equations. Nonlinear Anal. 7, 827-850 (1983) Zbl 0539.35027 MR 0709038

[9] Gilbarg, D., Trudinger, N. S.: Elliptic Partial Differential Equations of Second Order. Classics in Math. Springer, Berlin (2001) Zbl 1042.35002 MR 1814364

[10] Giusti, E.: Minimal Surfaces and Functions of Bounded Variation. Monogr. Math. 80, Birkhäuser, Basel (1984) Zbl 0545.49018 MR 0775682

[11] Gurtin, M. E.: On a theory of phase transitions with interfacial energy. Arch. Rat. Mech. Anal. 87, 187-212 (1985) MR 0768066

[12] Juutinen, P., Lindqvist, P., Manfredi, J.: On the equivalence of viscosity solutions and weak solutions for a quasilinear equation. SIAM J. Math. Anal. 33, 699-717 (2001) Zbl 0997.35022 MR 1871417

[13] Modica, L.: The gradient theory of phase transitions and the minimal interface criterion, Arch. Rat. Mech. Anal. 98, 123-142 (1987) Zbl 0616.76004 MR 0866718

[14] Modica, L., Mortola, S.: Un esempio di $\Gamma^{-}$-convergenza. Boll. Un. Mat. Ital. B (5) 14, 285-299 (1977) Zbl 0356.49008 MR 0445362

[15] Petrosyan, A., Valdinoci, E.: Geometric properties of Bernoulli-type minimizers. Interfaces Free Bound. 7, 55-77 (2005) Zbl pre02157849 MR 2126143

[16] Petrosyan, A., Valdinoci, E.: Density estimates for a degenerate/singular phase-transition model. SIAM J. Math. Anal. 36, 1057-1079 (2005)

[17] Rowlinson, J. S.: Translation of J. D. van der Waals' "The thermodynamic theory of capillarity under the hypothesis of a continuous variation of density". J. Statist. Phys. 20, 197-244 (1979) MR 0523642

[18] Savin, V. O.: Phase transitions: regularity of flat level sets. Ph.D. thesis at the University of Texas at Austin (2003)

[19] Tolksdorf, P.: On the Dirichlet problem for quasilinear equations in domains with conical boundary points. Comm. Partial Differential Equations 8, 773-817 (1983) Zbl 0515.35024 MR 0700735

[20] Tolksdorf, P.: Regularity for a more general class of quasilinear elliptic equations. J. Differential Equations 51, 126-150 (1984) Zbl 0488.35017 MR 0727034 
[21] Valdinoci, E.: Plane-like minimizers in periodic media: jet flows and Ginzburg-Landau-type functionals. J. Reine Angew. Math. 574, 147-185 (2004) Zbl pre02104132 MR 2099113

[22] Valdinoci, E., Sciunzi, B., Savin, V. O.: Flat level set regularity of $p$-Laplace phase transitions. Preprint

[23] Vázquez, J. L.: A strong maximum principle for some quasilinear elliptic equations. Appl. Math. Optim. 12, 191-202 (1984) Zbl 0561.35003 MR 0768629 